\title{
Cohesive cracked-hinge model for simulation of fracture in one-way slabs on grade
}

Skar, Asmus; Poulsen, Peter Noe; Olesen, John Forbes

Published in:

The International Journal of Pavement Engineering

Link to article, DOI:

10.1080/10298436.2017.1293263

Publication date:

2019

Document Version

Peer reviewed version

Link back to DTU Orbit

Citation (APA):

Skar, A., Poulsen, P. N., \& Olesen, J. F. (2019). Cohesive cracked-hinge model for simulation of fracture in oneway slabs on grade. The International Journal of Pavement Engineering, 20(3), 298-302.

https://doi.org/10.1080/10298436.2017.1293263

\section{General rights}

Copyright and moral rights for the publications made accessible in the public portal are retained by the authors and/or other copyright owners and it is a condition of accessing publications that users recognise and abide by the legal requirements associated with these rights.

- Users may download and print one copy of any publication from the public portal for the purpose of private study or research.

- You may not further distribute the material or use it for any profit-making activity or commercial gain

- You may freely distribute the URL identifying the publication in the public portal

If you believe that this document breaches copyright please contact us providing details, and we will remove access to the work immediately and investigate your claim. 
To appear in the International Journal of Pavement Engineering

Vol. 00, No. 00, 00 Month 20XX, 1-25

\title{
Research article
}

\section{Cohesive cracked-hinge model for simulation of fracture in one-way slabs on grade}

(December 9, 2016)

\begin{abstract}
Numerical analysis of slab on grade structures subjected to mechanical loads is a complex matter often requiring computationally expensive models. In order to develop a simplified and general concept for non-linear analysis of slab on grade structures, this paper presents a cohesive cracked-hinge model aimed at the analysis of the bending fracture of the cemented material. The model is based on the fracture mechanics concepts of the fictitious crack model with a linear stress-crack opening relationship. Moreover, the paper presents a two-parameter spring foundation model applied to realistically capture the continuity in the supporting medium. The functionality of the proposed model is compared to numerical analysis with application of the more conventional cohesive zone model. The results obtained show that the methodology is attractive and powerful one well-suited for practical use and further development.
\end{abstract}

Keywords: Cohesive crack; non-linear FEM; slab on grade; cemented materials; pavement analysis; two-parameter foundation model

\section{Introduction}

Numerical analysis of fracture in concrete- and composite pavement systems, or so-called slab on grade structures, is a highly complex matter. This type of analysis often require large- and computationally expensive models applicable to relatively simple design problems. Moreover, concrete- and composite pavement systems typically exhibit softening load-displacement postpeak behaviour in bending on both material- and structural level. This often results in convergence issues and aborted simulations making complex non-linear analysis less attractive for design engineering purposes. In order to create a simple and robust modeling framework for engineering application, this paper presents a non-linear cracked-hinge model based on the fracture mechanics concepts of the fictitious crack model (Hillerborg et al. 1976). The hinge model for modeling the crack propagation due to pure bending in a concrete beam without reinforcement was first presented by Ulfkjær et al. (1995). This hinge was successfully applied in the modeling of pure concrete beams in three point bending considering the development of only one crack. Olesen (2001b) expanded the hinge model by applying a bi-linear softening curve to allow for the incorporation of the effects of fibers on concrete fracture. Further, this modified hinge model allowed for the existence of a sectional normal force. This last feature is crucial for the ability of the hinge to model a number of situations such as the wedge splitting test (Walter et al. 2005) and the split cylinder test (Olesen et al. 2006). Subsequently, this type of semi-analytical hinge models have primarily been applied for analysis of fracture in reinforced concrete beams (Olesen 2001a; Kwak and Kim 2002, 2010; Buratti et al. 2011; Carpinteri and Corrado 2011; Castel et al. 2011; Visintin et al. 2012). Murthy et al. (2013) used a hinge model to evaluate bi-linear softening diagrams of plain concrete corresponding to their size independent fracture energy and Wardeh and Ghorbel (2015) used a hinge model to study the effect of frost action on fracture properties and strain softening behavior.

The principles of the fictitious crack model have been extended to practical problems for concrete- and composite pavement structures applying cohesive interface elements or contact for- 
mulations, with some very encouraging results (Meda et al. 2004; Ioannides et al. 2006; Gaedicke and Roesler 2009; Gaedicke et al. 2012; Aure and Ioannides 2012; Evangelista et al. 2013; Aure and Ioannides 2015; Skar and Poulsen 2015). Gaedicke and Roesler (2009) and Gaedicke et al. (2012), who published one of the few simulations with experimental results, used the interface element with tabular traction-displacement relation featured by ABAQUS (2013). The authors reported lack of convergence, instability problems, snap-backs, and aborted simulations upon local unloading and reloading paths. Aure and Ioannides (2012) presented reduced finite element models compared to Gaedicke et al. and found, that for slabs on grade structures, the type of softening curve, cohesive zone width and mesh do not influence the response significantly. Evangelista et al. (2013) developed a user-built cohesive element based on a damage mechanics framework resulting in more robust cohesive elements. Skar and Poulsen (2015) used the cohesive surface model in ABAQUS and applied a relatively coarse mesh for the cohesive zone. This resulted in computationally efficient models for analysis of the composite pavement systems studied. However, although there are successful examples of optimisation with regard to model size and robustness, all methods published deal with relatively large finite element models compared to the complexity of the pavement system studied.

Another issue is the idealisation of soil foundation properties often applied in analysis of slab on grade structures. The interaction between structure and foundation and soil foundation properties are commonly idealised as independent linear elastic springs or so-called Winklers foundation (Winkler 1868). The Winkler model is simple and practical to many engineering problems, however, care should be taken in application of such model as it essentially suffers from a complete lack of continuity in the supporting medium. Moreover, another fundamental problem with the use of this model is to determine the stiffness of elastic springs used to replace the soil. The problem becomes two-fold since the numerical value of the Winkler stiffness (or modulus of subgrade reaction) not only depends on the nature of the soil foundation, but also on the dimensions of the slab and the loaded area.

Traditionally, the way to overcome the deficiency of Winkler models is to introduce an interaction between the independent springs, e.g., interconnections such as flexural elements, shear layers and deformed, pre-tensioned membranes (Kerr 1964; Hetenyi 1966). This class of mathematical models have another constant parameter which characterises the interaction implied between springs and hence are called two-parameter foundation models. However, interpretation of how soil foundation material properties and characteristics are reflected in the various elements in the mechanical foundation models can be difficult, thus evaluation on a rational, theoretical basis is cumbersome.

The semi-analytical hinge models reported in the literature are effective for studying the behavior of simple fracture tests or problems where the crack path is known a-priori. However, for studying more complex problems, a numerical formulation of the hinge is more convenient. Thus, this study presents a finite element (FE) cohesive cracked-hinge beam resting on a twoparameter foundation for analysis of fracture in one-way slabs on grade supported by an elastic medium. At the lowest level we consider a strip of cemented material including a crack and establish a stress-mean strain relationship. At the intermediate level we consider a hinge element which is a finite part of the beam consisting of layered strips of cemented material, and establish a relationship between generalised sectional forces and strains. At the highest level we apply the hinge model as a constitutive model in a non-linear beam element.

The effective stiffness concept is applied allowing formation of multiple cracks along the slab axis which is an essential feature of both plain- and reinforced concrete slabs before exhibiting localised rotations. The effective stiffness which is a function of the state of deformation is treated as a constitutive relationship. Although the underlying description of the hinge is based on the formation of discrete cracks the constitutive behavior of the hinge is smeared (smooth). This particular feature is practical and effective as it requires no a-priori knowledge of the crack pattern.

We show how the cracked-hinge model can be extended to pavement applications, imple- 
menting a two-parameter foundation model into beam elements. Moreover, a simple method for estimating foundation model parameters is proposed. This part is important because it describes a simplified methodology for simulating fracture in slabs on grade structures linking all necessary analysis steps in a rational and consistent manner.

The objective of the work presented is to develop a general and consistent framework based on a mechanistic approach for design of concrete- and composite pavements. In this paper we focus on establishing a simple model for two-dimensional analysis, assuming a continuum representation of the soil medium. The general and consistent format selected makes the model suited for further developments, taking into account more complex material behavior, soil response and cyclic loading conditions.

\section{Methodology}

\subsection{The mechanics of the cracked-hinge model}

The basic assumption of the hinge model is the fact that the presence of a crack influences the overall stress and strain field of a structure only locally. The discontinuity created by the crack is expected to vanish outside a certain width. Under constant moment, e.g. between the loaded points in Figure 1 (a), the beam sections at the midpoints between the cracks will, due to the periodicity of the cracks, remain plane during deformation of the beam. The width $s$ between two such sections embracing one crack defines a hinge element, as shown in Figure 1 (b). For the beam area outside the loaded points, the moment distribution is no longer constant. Such phenomena can be handled with appropriate numerical tools, i.e. the finite element (FE) method, as exemplified for a single beam element in Figure 1 (b).

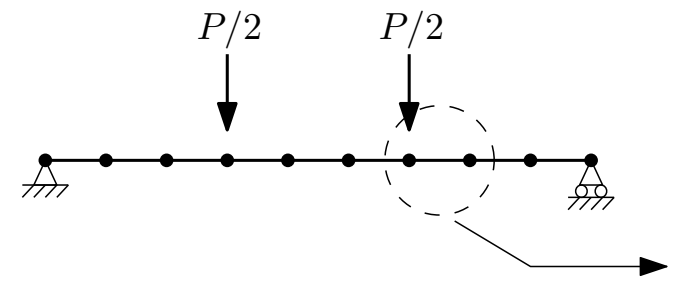

(a)

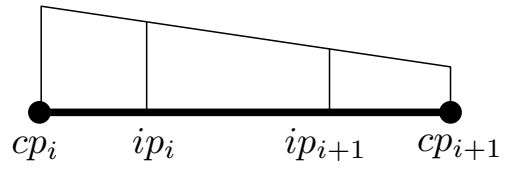

$\mathbf{\nabla}---------$

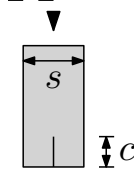

(b)

Figure 1. Sketch of hinge model implemented in simply supported beam under four point loading: (a) overview of beam structure, (b) underlying discrete formulation of cracks at constitutive points, $c p$, and smeared constitutive behavior obtained from interpolation between constitutive points at integration points, $i p$.

The hinge width $s$ is a fundamental calibration parameter of the model, and it was suggested in Ulfkjær et al. (1995) to use a hinge width half the height of the beam, also adopted in the present study. Thus, the flexural deformation of the beam is concentrated and the propagation of a crack can be modelled as a hinge whereas the rest of the beam can be treated as elastic bulk material.

The uni-axial tensile behaviour of the concrete is modeled according to the fictitious crack model by Hillerborg et al. (1976). The linear elastic pre-crack state is described by the elastic modulus, $E_{c}$. The uni-axial tensile strength is denoted by $f_{t}$ and the corresponding strain by $\varepsilon_{c t}$. For the fracture analysis of concrete slab on grade pavement structures the stress-crack opening relationship, or so-called softening law, does not influence the response significantly (Gaedicke and Roesler 2009; Aure and Ioannides 2012). Thus, in the present study the softening law is given as a linear curve 


$$
\sigma(w)=f_{t}+a w
$$

where $a$ is the negative gradient on the softening curve and $w$ is the crack opening. The fracture energy, $G_{F}$, is given by the area under the softening curve, resulting in a final zero-stress crack opening $w_{c}=-f_{t} / a$.

The hinge model provides the relationship between the state of deformation of the hinge and the sectional forces $(N, M)$. The state of deformation is described by the mean normal strain of the beam axis and the mean curvature of the hinge, $\left(\bar{\varepsilon}_{0}, \bar{\kappa}\right)$.

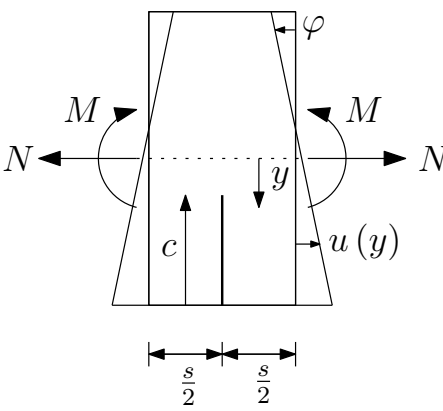

(a)

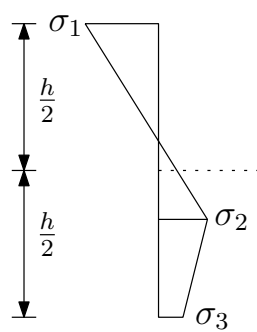

(b)

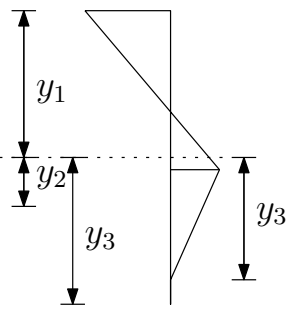

(c)

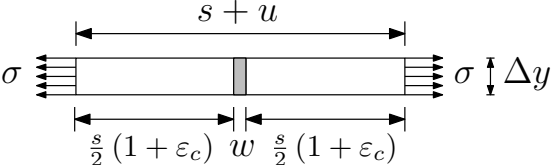

(d)

Figure 2. Hinge model: a) beam segment with constant sectional forces and deformation of cracked beam segment. b) and c) hinge stress distribution after initiation of cracking at the two different Phases $I$ and $I I$, respectively. The distances $y_{1}$ to $y_{3}$ is used to perform integration in blocks shown in Appendix B. (d) material strip in uni-axial tension: loaded state beyond peak-load showing crack deformations.

The hinge solution is based on the assumption that the hinge may be seen as consisting of independent infinitesimal layers of cemented material. The tensile behaviour of this layer may be established by considering a strip of material in uni-axial tension as, shown in Figure 2 (d). The elongation of the strip located at $y$ can be expressed in terms of the mean normal strain

$$
\bar{\varepsilon}(y)=\frac{2 u(y)}{s}=\frac{2 u_{0}(y)}{s}+\frac{2 \varphi}{s} y \equiv \bar{\varepsilon}_{0}+\bar{\kappa} y
$$

where $\bar{\varepsilon}_{0}$ is the mean normal strain at the beam axis, and $\bar{\kappa}$ the mean curvature of the hinge. Utilizing (2) the depth coordinates of characteristic points of the stress distribution at the midsection of the hinge may be determined.

Assume that the hinge has been deformed to a state where a crack has formed and penetrated a distance $c$ into the hinge. The position of the crack tip is denoted by $y_{2}=h / 2-c$, and is given as follows

$$
\sigma_{2}=f_{t} \Rightarrow\left(\bar{\varepsilon}_{0}+\bar{\kappa} y_{2}\right) E_{c} \Rightarrow y_{2}=\frac{\varepsilon_{c r}-\bar{\varepsilon}_{0}}{\bar{\kappa}}
$$

In the cracked state, $0<w \leq w_{c}$ (Phase $I$ ), the crack opening and the corresponding stress in the strip is given as

$$
\left.\begin{array}{l}
\sigma_{c}=\sigma_{w} \Rightarrow \sigma_{c}=f_{t}+a w \\
s+2 u=s\left(1+\varepsilon_{c}\right)+w \Rightarrow s \bar{\varepsilon}(y)=s \varepsilon_{c}+w
\end{array}\right\} \Rightarrow\left\{\begin{array}{l}
w^{I}=s \frac{E_{c} \bar{\varepsilon}(y)-f_{t}}{E_{c}+a s} \\
\sigma_{3}^{I}=E_{c} \frac{f_{t}-a s \bar{\varepsilon}(y)}{E_{c}-a s}
\end{array}\right.
$$

In the cracked state, $w_{c} \leq w$ (Phase $\left.I I\right)$, the crack is stress free, leading to the simple solution for the strip 


$$
\left.\begin{array}{l}
w=s(1+\bar{\varepsilon}(y))-s \\
\sigma_{c}=0
\end{array}\right\} \Rightarrow\left\{\begin{array}{l}
w^{I I}=s \bar{\varepsilon}(y) \\
\sigma_{3}^{I I}=0
\end{array}\right.
$$

From (4) and (5) the state of stress and crack opening in the cracked part of the hinge can be established. The cohesive stresses extend from $y=y_{2}$ to $y=y_{3}$, depicted in Figure 2 (b) and (c); $y_{2}$ is given by (3) and

$$
y_{3}=\min \left(\frac{h}{2},-\frac{1}{\bar{\kappa}}\left(\frac{f_{t}}{a s}+\varepsilon_{0}\right)\right)
$$

Finally, the sectional forces with respect to $y=0$ may then be calculated from integration over the hinge height

$$
\begin{gathered}
N\left(\bar{\varepsilon}_{0}, \bar{\kappa}\right)=t \int_{-h / 2}^{h / 2} \sigma_{c} d y \\
M\left(\bar{\varepsilon}_{0}, \bar{\kappa}\right)=t \int_{-h / 2}^{h / 2} \sigma_{c} y d y
\end{gathered}
$$

Contribution from a reinforcement bar can be included by adding the term $A_{s} \sigma_{s}$ and $A_{s} \sigma_{s} y_{s}$, in (7a) and (7b), respectively. Where $A_{s}$ is the area, $\sigma_{s}$ is the stress in the steel- and $y_{s}$ is the position of the rebar.

\section{$2.2 \quad$ Foundation model and calibration}

\subsubsection{The mechanics of the two-parameter foundation model}

The development of two-parameter models has been approached along two distinct lines. The first type proceeds from the discontinuous Winkler model and eliminates its discontinuous behavior by providing mechanical interaction between the spring elements by either elastic membranes (Filonenko-Borodich 1940), elastic beams (Hetényi 1946) or elastic layers capable of pure shear deformation (Pasternak 1954; Kerr 1964). The second approach starts from the elastic continuum model and simplifying assumptions with respect to the distribution of displacements and stresses, see e.g. Reissner (1958); Vlasov (1966).

The influence of using two-parameter models in design of rigid pavement slab on grade structures have been studied by e.g. Ioannides et al. (1985) and Khazanovich and Ioannides (1993), following implementation of such models in several special purpose finite element (FE) tools for rigid pavement design (NCHRP 2003). Moreover, two-parameter models have been used to study complex phenomena occurring in rigid pavements, such as slab-curling (Khazanovich and Ioannides 1994; Khazanovich 2003; Zokaei-Ashtiani et al. 2015) and layer contact problems (Zokaei-Ashtiani et al. 2014). However, the influence of using two-parameter models, or soil-idealisation techniques in general, for analysis of slab fracture has drawn little attention.

Several recent efforts have been made to implement mechanical models more sound and logic than the Winkler model. However, no foundation model has yet replaced the Winkler model and 
achieved a reasonably widespread level of acceptance among design engineers (Horvath 2002; Colasanti and Horvath 2010). In this aspect, the Winkler foundation with shear interaction is mechanically a logical extension of the Winkler model and analytically the next higher approximation (Kerr 1965). This model offers an attractive alternative to the elastic solid continuum by providing a degree of shear interaction between adjacent soil elements, while remaining relatively simple to analyse (Ioannides 2006). Moreover, implementation of such a model in commercial $\mathrm{FE}$ codes is straightforward using discrete spring elements.

The two-parameter foundation model presented here is composed of coupled spring elements similar to the methodology proposed by Loof (1965). The coupling between two springs to ground is modeled as second spring transmitting a shear force $T$ per unit width, as shown in Figure 3 (a). This force is associated with the difference in vertical displacements $w$ between the elements.

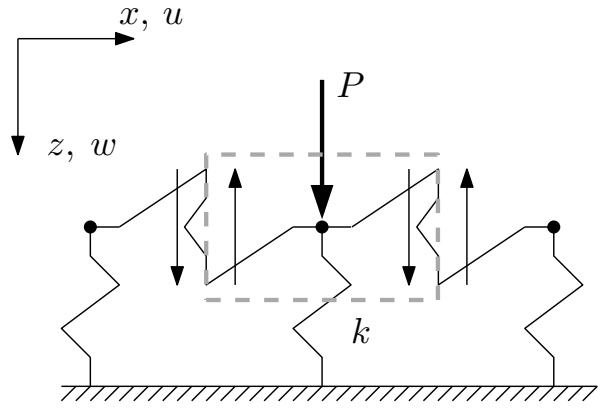

(a)

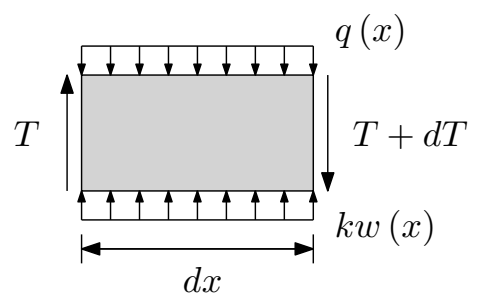

(b)

Figure 3. Mechanical model of two-parameter spring foundation, where $k$ is the Winkler stiffness and $\gamma$ is the second parameter stiffness.

A simple assumption is that the shear force is proportional to the difference in displacements between two consecutive elements and therefore to the first derivative of the displacement, where $\gamma$ is the second parameter and equivalent to the shear constant $G_{p}$ in the Pasternak model. From the equilibrium of an element, shown in Figure 3 (b), the differential equation governing the deflections of the soil surface is established as

$$
q(x)=k w(x)-\frac{d T}{d x} \Rightarrow q(x)=k w(x)-\gamma \frac{d^{2}}{d x^{2}} w(x)
$$

where the Winkler stiffness is $k=k_{0} t, k_{0}$ is the modulus of subgrade reaction and $t$ is the plane strain thickness of the model.

First, the case of a point load $P$ is investigated, shown in Figure 4 (a). Introducing the terms $\alpha^{2}=\gamma / k$ and $\beta=1 / \alpha$ the homogeneous solution yields

$$
w(x)=C_{1} e^{-\beta x}+C_{2} e^{\beta x}
$$

In the solution for large positive values of $x$ in (9) the term with the coefficient $C_{1}$ represents the decreasing displacement due to the influence of the load. The solution for positive $x$ can be found, inserting the boundary condition $w \rightarrow 0$ for $x \rightarrow \infty$ in (9), i.e. $w(x \rightarrow \infty): C_{1} e^{-\beta x} \rightarrow 0$ and $C_{2} e^{\beta x} \rightarrow \infty \Rightarrow C_{2}=0$. The constant $C_{1}$ can now be found, assuming that the difference in shear force to the right due to the point load in $x=0$ is equal to half the magnitude of the point load, giving the relations; $\Delta T=P / 2, \Delta T=\gamma \Delta w^{\prime}=\Delta T=\gamma w^{\prime}(0)$ and $w^{\prime}(0)=-\beta C_{1} e^{-\beta \cdot 0}=-\beta C_{1}$. Combining these relations and applying the term $\alpha^{2}=\gamma / k$ the solution for a point load is given as 


$$
w_{P}(x)=\frac{P}{2 k \alpha} e^{-\beta x}
$$

Using the superposition technique, (10) can be integrated to obtain expressions for the surface

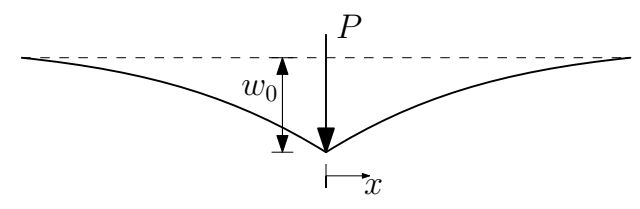

(a)

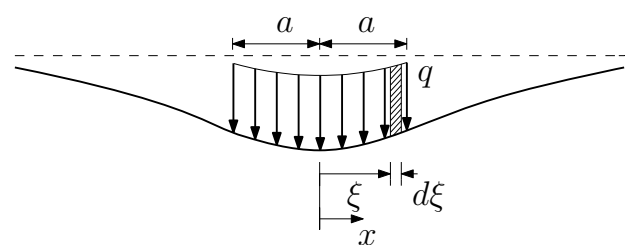

(b) ${ }^{x}$

Figure 4. Sketch of elastic isotropic continuum foundation subjected to (a) a point load $P$ and (b) a distributed load $q$.

displacements for a uniformly distributed load shown in Figure 4 (b), given as

$$
\begin{aligned}
& w_{q}(x)=\frac{q}{2 k}\left[2-e^{-\beta(x+a)}-e^{-\beta(a-x)}\right] \quad \text { for } x \leq|a| \\
& w_{q}(x)=-\frac{q}{2 k}\left[e^{-\beta(x+a)}-e^{-\beta(a-x)}\right] \quad \text { for } x \geq|a|
\end{aligned}
$$

where $x \leq|a|$ and $x \geq|a|$ symbolises if the displacement is calculated inside or outside the loaded area, respectively.

\subsubsection{Calibration of spring parameters}

In order to obtain the necessary foundation model parameters this study presents a simple methodology, combining the two-parameter model presented in Section 2.2.1, and the elastic continuum theory. The problem of the elastic stress field within a semi-infinite medium loaded by a point load has been solved by Flamant (1892) using Boussinesq's solution (Timoshenko et al. 1970). The surface displacement in the vertical direction can be written as

$$
w_{P}(x)=\frac{2 P}{\pi E} \ln \left(\frac{d}{|x|}\right)-\frac{(1-\nu) P}{\pi E}
$$

where $P$ is the point load, $E=\frac{E_{s}}{\left(1-\nu_{s}^{2}\right)}$ and $\nu=\frac{\nu_{s}}{\left(1-\nu_{s}\right)}$ is the Young's modulus and Poisson's ratio of the soil foundation, respectively, $|x|$ is the numerical value of $x$ (distance from the loaded point) and $d$ is a rigid body constant found from assuming zero vertical displacements at a distance $x=d$.

Using the superposition technique, (12) can be integrated to obtain expressions for the surface displacements for a uniformly distributed load, given as

$$
\begin{aligned}
w_{q}(x) & =-\frac{2 q}{\pi E}\left[(x-a) \ln \left(\frac{d}{|a-x|}\right)+(-a-x) \ln \left(\frac{d}{|x+a|}\right)\right. \\
& +a(\nu-1)] \text { for } x \leq|a|
\end{aligned}
$$




$$
\begin{aligned}
w_{q}(x) & =-\frac{2 q}{\pi E}\left[(x-a) \ln \left(-\frac{d}{a-x}\right)+(-a-x) \ln \left(\frac{d}{|x+a|}\right)\right. \\
& +a(\nu-1)] \text { for } x \geq|a|
\end{aligned}
$$

where $x \leq|a|$ and $x \geq|a|$ symbolises if the displacement is calculated inside or outside the loaded area, respectively.

In the present study, the response of the slab and the influence of model parameters on the crack initiation and propagation is of primary interest. In this aspect the vertical displacements and the curvature of the foundation are important features due to localisation of cracks and the increasing soil stresses near the crack front during progressive cracking.

In order to realistically capture the response of an elastic continuum it is here proposed to calibrate the model analytically, combining these measures at different positions below the slab, i.e. the vertical displacement at the center of the loaded area $w(0)$, the vertical displacement at the edge of the loaded area $w(a)$ and the curvature at the center of the loaded area $w^{\prime \prime}(0)$. Thus, the continuum model and the two-parameter model yields the exact same result at these positions for each measure. Two-and-two measures are combined, resulting in three different analytical calibration methods. The relevant expressions for the two model types, i.e. $w(0), w(a)$ and $w^{\prime \prime}(0)$, are given in Appendix A.1 and A.2.

The two-parameter model can now be calibrated; first, the theoretical displacements and curvature at the centre and displacements at the edge of the distributed load can be found from (A2a)-(A2c) and inserted in the relevant expressions for the two-parameter model, i.e. (A1a)(A1c). A simple procedure is adopted here keeping one parameter constant, e.g. for calibration method no. 1 , we have $\gamma \rightarrow \gamma_{w_{0}}=\gamma_{w_{0}^{\prime \prime}}$. The equation is then solved iteratively for $k$ until $k_{w_{0}}=k_{w_{0}^{\prime \prime}}$.

Table 1. Calibrated foundation model parameters $k$ and $\gamma$

\begin{tabular}{cccccc}
\hline Method & Criteria & $k\left(\mathrm{~N} / \mathrm{mm}^{2}\right)$ & $\gamma(\mathrm{N})$ & $R^{2}(\mathrm{x} \leq 10 \mathrm{~m})$ & $R^{2}(\mathrm{x} \leq 1.0 \mathrm{~m})$ \\
\hline 1. & $w_{0} \& w_{0}^{\prime \prime}$ & 0.0076 & $6.33 \cdot 10^{7}$ & 0.976 & 0.999 \\
2. & $w_{0} \& w_{a}$ & 0.0104 & $3.95 \cdot 10^{7}$ & 0.928 & 1.000 \\
3. & $w_{0}^{\prime \prime} \& w_{a}$ & 0.0086 & $6.18 \cdot 10^{7}$ & 0.978 & 0.992 \\
\hline
\end{tabular}

In order to evaluate the influence of the different calibration methods a uniformly distributed load over a strip length $l$ of $2.0 \mathrm{~m}$ is applied directly on the soil surface comparing the twoparameter model and the elastic continuum model. Due to symmetry conditions only half the loaded strip $a=l / 2$ is considered here. The resulting calibrated foundation model parameters can be found in Table 1.

Comparing the surface displacements for the continuum model and the three different twoparameter models, shown in Figure 5 (a) and Table 1, it is observed that Calibration Method no. 1 gives a good overall fit. Method no. 2 gives the closest prediction of the surface displacements in the loaded region, i.e. $x \leq 1.0 \mathrm{~m}$, whereas method no. 3 yields realistic results far from the loaded region. It is also observed from Figure 5 (b) that increasing the load strip length results in a decreased $k$, stabilising at app. $a=1-2 \mathrm{~m}$. Whereas $\gamma$ increases with increasing load strip length, stabilising at app. $a=4-5 \mathrm{~m}$.

\subsection{Implementation of hinge- and foundation model into beam element}

The hinge- and two-parameter model is implemented following standard finite element beam theory and procedures for building elements with non-linear material behaviour, see e.g. Cook et al. (2007). The expressions for the element stiffness matrix and equivalent nodal loads are based on the cubic displacement function and for direct incorporation of the foundation model. Thus, full contact is assumed between the beam and the foundation. 


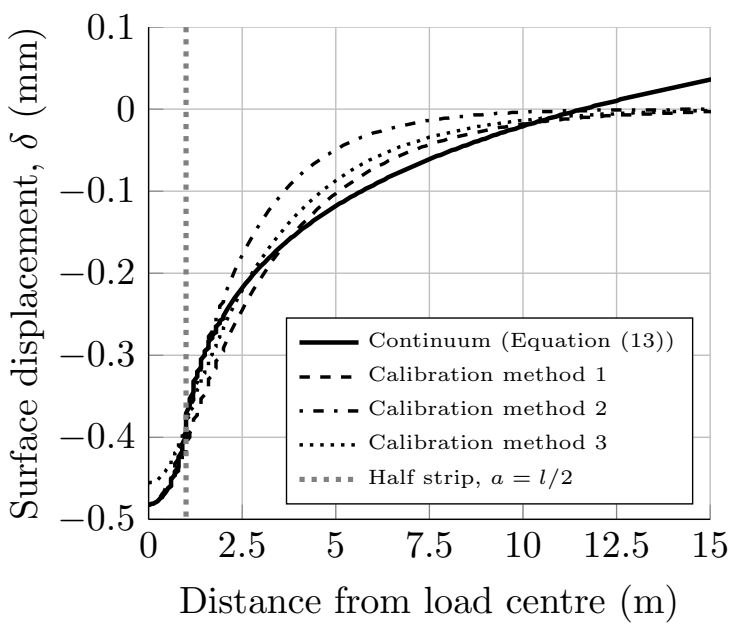

(a)

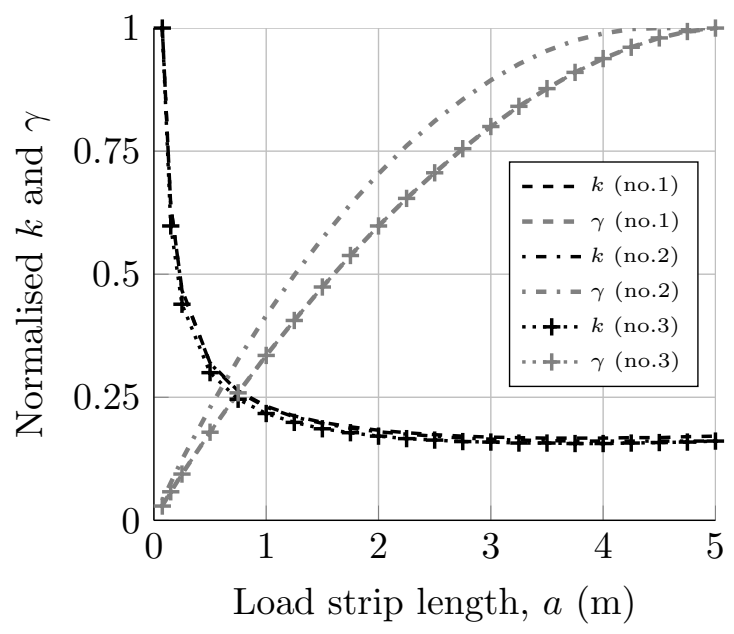

(b)

Figure 5. (a) Comparison of analytical and two-parameter foundation model for the three different calibration techniques (b) influence of half load strip length $a$ on model parameters $k$ and $\gamma$ for a distributed load (normalised with respect to maximum value obtained in interval $0-5 \mathrm{~m}$ ). Uniformly distributed load: $q=25,000 \mathrm{~N} / \mathrm{m}$. Soil properties: $E_{s}=100 \mathrm{MPa}$, $\nu_{s}=0.35, t=1 \mathrm{~m}$ and $d=25 \mathrm{~m}$.

For the present study a plane three-node beam element is chosen as shown in Figure 6 . This element is capable of modeling quadratic variations of the axial displacements and cubic variations of the transverse displacements. The choice of element ensures that both generalised strains are interpolated linearly as opposed to a typical two-node beam element where constant normal strain is assumed.

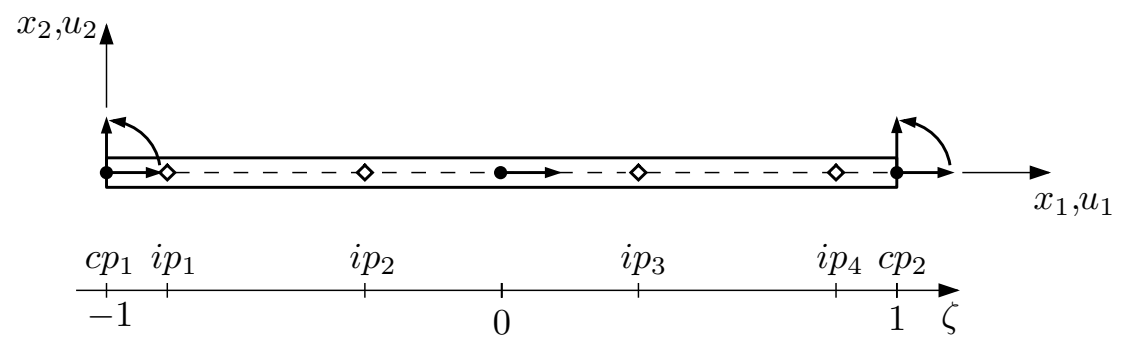

Figure 6. Plane beam element: Constitutive points $\left(c p_{i}\right)$ are located at endpoints, integration points $\left(i p_{i}\right)$ at Gauss points $(i=1,4) \pm 0.861136312$ and $(i=2,3) \pm 0.339981044$ ( $6^{\text {th }}$ order polynomial function).

The vector of generalised displacements of the beam, $\boldsymbol{u}$, holds the axial displacements $u_{1}\left(x_{1}\right)$ and the transverse displacements $u_{2}\left(x_{2}\right)$. The interpolation of $\boldsymbol{u}$ in the element is given by

$$
\boldsymbol{u}=\left[\begin{array}{l}
u_{1} \\
u_{2}
\end{array}\right]=\mathbf{N} \boldsymbol{v}
$$

where $\mathbf{N}$ is the displacement interpolation matrix and $\boldsymbol{v}$ is the element dof vector. The interpolation functions are standard polynomial expressions. The vector of generalised strains, $\varepsilon$, holds the linearised axial strain $\varepsilon_{0}$ and the linearised curvature $\kappa$. The interpolation of $\varepsilon$ in the element is given by 


$$
\varepsilon=\left[\begin{array}{c}
\varepsilon_{0} \\
\kappa
\end{array}\right]=\left[\begin{array}{c}
\frac{d u_{1}}{d x} \\
\frac{d^{2} u_{2}}{d x^{2}}
\end{array}\right]=\mathbf{B} \boldsymbol{v}
$$

where $\mathbf{B}$ is the strain interpolation matrix. The vector of generalised stresses, $\boldsymbol{\sigma}$, holds the sectional normal force $N$ and the sectional moment $M$. In the element $\boldsymbol{\sigma}$ may be established applying (7a) and (7b) as

$$
\boldsymbol{\sigma}=\boldsymbol{\sigma}(\varepsilon)=\left[\begin{array}{l}
N(\varepsilon) \\
M(\varepsilon)
\end{array}\right]
$$

Based on the contribution to the variation in internal work $\delta \Omega$, from the beam and the twoparameter foundation, and the potential work of external forces $\delta W$ from point- and surface loads, the principle of virtual work for the system can be established

$$
\int_{V} \delta \boldsymbol{\varepsilon}^{T} \boldsymbol{\sigma}+\delta \boldsymbol{u}^{T} k \boldsymbol{u}+\delta \boldsymbol{\theta}^{T} \gamma \boldsymbol{\theta} d V=\int_{S} \delta \boldsymbol{u}^{T} \boldsymbol{f} d S+\sum_{i} \delta \boldsymbol{u}_{i}^{T} p_{i}
$$

where $V$ is the structural volume, $S$ is the surface area, $\delta \boldsymbol{u}$ and $\delta \boldsymbol{\theta}$ is the displacement and rotational variations, respectively, $\boldsymbol{f}$ is the surface traction vector, $p_{i}$ is a concentrated (nodal) load and $\delta \boldsymbol{u}_{i}$ is the associated (nodal) displacement variation. The contribution of the second parameter spring is here given on a general form considering $\gamma$ as a rotational stiffness.

The beam-element and two-parameter foundation contribution to the internal nodal force, $\boldsymbol{q}$, is then given by the expression

$$
\boldsymbol{q}=\int_{0}^{L_{e}} \mathbf{B}^{T} \boldsymbol{\sigma} d x+\int_{0}^{L_{e}} \mathbf{N}^{T} k \mathbf{I N} \boldsymbol{v}_{e} d x+\int_{0}^{L_{e}} \mathbf{G}^{T} \gamma \mathbf{I} \mathbf{G} \boldsymbol{v}_{e} d x
$$

where $L_{e}$ is the length of the element and $\boldsymbol{v}_{e}$ is the global dof element displacements. The matrix $\mathbf{I}=\left[\begin{array}{ll}0 & 0 \\ 0 & 1\end{array}\right]$ is used to omit axial terms in interpolation matrix $\mathbf{N}$ and $\mathbf{G}$. The latter is given as $\mathbf{G}=\mathbf{N}^{\prime}$.

The beam-element and two-parameter foundation contribution to the tangential stiffness matrix, $\mathbf{k}_{t}$, is given by the expression

$$
\mathbf{k}_{t}=\int_{0}^{L_{e}} \mathbf{B}^{T} \mathbf{D}_{t} \mathbf{B} d x+\int_{0}^{L_{e}} \mathbf{N}^{T} k \mathbf{I N} d x+\int_{0}^{L_{e}} \mathbf{G}^{T} \gamma \mathbf{I} \mathbf{G} d x
$$

The hinge tangent stiffness matrix, $\mathbf{D}_{t}$ is defined through

$$
\left[\begin{array}{l}
d N \\
d M
\end{array}\right]=\mathbf{D}_{t}\left[\begin{array}{l}
d \bar{\varepsilon}_{0} \\
d \bar{\kappa}
\end{array}\right], \text { where } \mathbf{D}_{t}=\left[\begin{array}{ll}
\frac{\partial N}{\partial \bar{\varepsilon}_{0}} & \frac{\partial N}{\partial \bar{\kappa}} \\
\frac{\partial M}{\partial \bar{\varepsilon}_{0}} & \frac{\partial M}{\partial \bar{\kappa}}
\end{array}\right]
$$


Full derivation of the hinge tangent stiffness matrix, $\mathbf{D}_{t}$, can be found in Appendix B.

The hinge model is implemented in a user-built finite element code using the numerical computing package MATLAB. The functionality of the proposed hinge is tested for a simply supported beam under four point loading and compared with experimental- and numerical studies on cement bound granular mixtures reported in Yeo (2008) and Skar and Poulsen (2015). Comparison of experimental- and numerical results is evaluated in the view of load-displacement and load-crack mouth opening displacement (CMOD) behavior, shown in Figure 7 (a) and (b), respectively. The load-displacement response is given as the displacement under the loaded point, or so-called load line displacement (LLD).

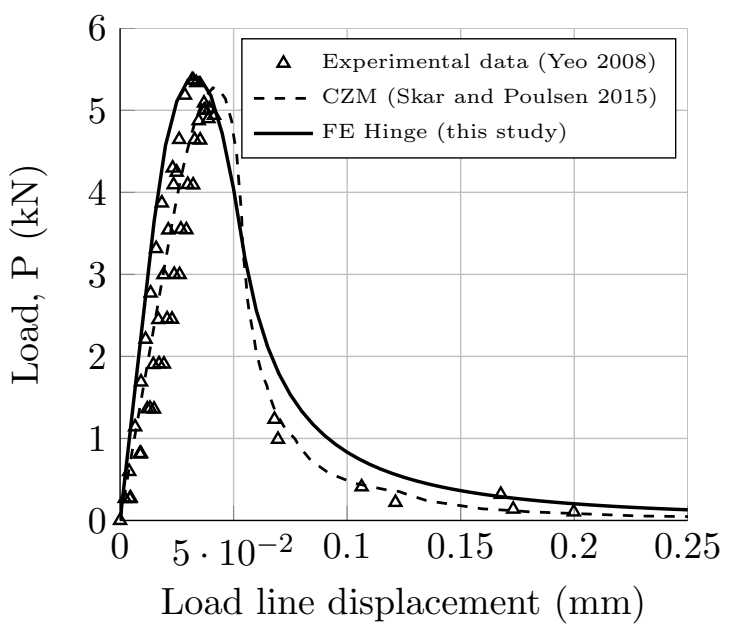

(a)

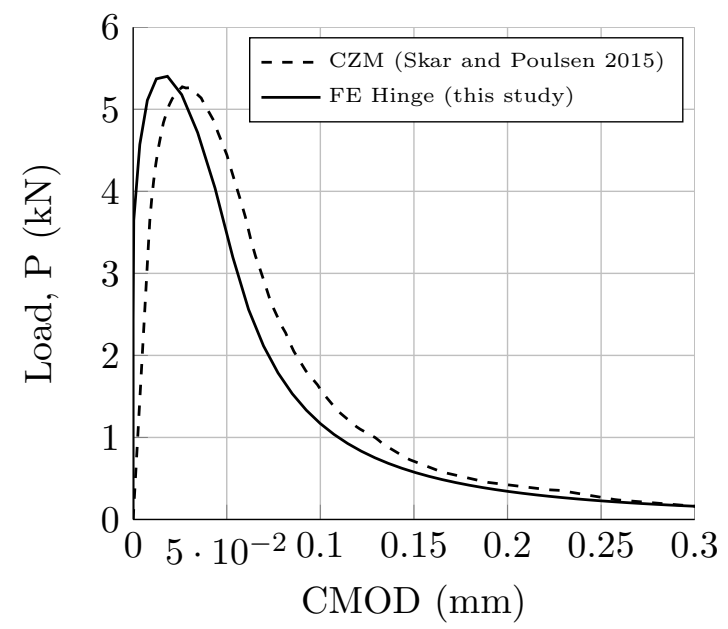

(b)

Figure 7. Comparison between the proposed hinge and experimental- and numerical results reported in Yeo (2008) and Skar and Poulsen (2015), respectively: (a) Load-displacement response (b) numerical Load-CMOD response. Beam geometry $(L / h / t): 300 \times 100 \times 100 \mathrm{~mm}^{3}$. Element size $9.375 \mathrm{~mm}$. Load positions: $L / 3,2 L / 3$. Mechanical properties: $E_{c}=12,760$ $\mathrm{MPa}, \nu=0.2, f_{t}=1.0 \mathrm{MPa}, G_{F}=0.028 \mathrm{~N} / \mathrm{mm}$.

It is observed from Figure 7 that there is good agreement between the proposed hinge model and experimental- and numerical results reported in the literature. The difference between numerical models, i.e. the cohesive zone model ('CZM') and the cracked-hinge model ('FE hinge'), is mainly related to the pre-peak behavior. Whereas the the hinge model behaves perfectly elastic up to initiation of cracking (load level of app. $3.5 \mathrm{kN}$ ), the cohesive zone model incorporates a small error in crack-opening displacements related to the penalty stiffness. This error is best exemplified in Figure 7 (b), observing that the crack-opening in the cohesive zone model evolves from the beginning of the analysis and thus resulting in a more flexible behavior of the beam. Increasing penalty stiffness reduce this error, however, very high levels of penalty stiffness result in ill-conditioned stiffness matrix and thus slow convergence and aborted simulations. This problem is avoided applying the finite element hinge, resulting in a more robust model and stable simulations.

\subsection{Continuum model, solution technique and numerical characteristics}

\subsubsection{Cohesive zone model resting on elastic medium}

In order to evaluate the hinge slab model and to investigate the influence of different modeling techniques for slab-soil interaction in concrete- and composite pavement systems a model representing the full continuum model is developed. A simplified two layer model is considered; a single slab, with standard dimensions $(L / h)$ of $4 \times 0.4 \mathrm{~m}^{2}$, constructed over soil foundation with 
rigid body constraint, $d=25 \mathrm{~m}$, representing the semi-infinite elastic medium, as shown in the sketch in Figure 8 (a). The slab is in subsequent sections referred to as the 'CZM slab'.

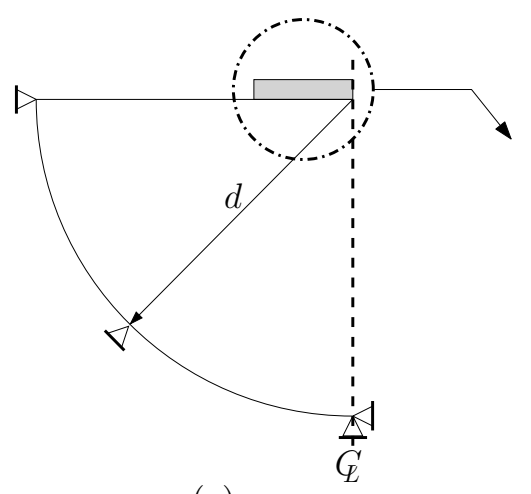

(a)

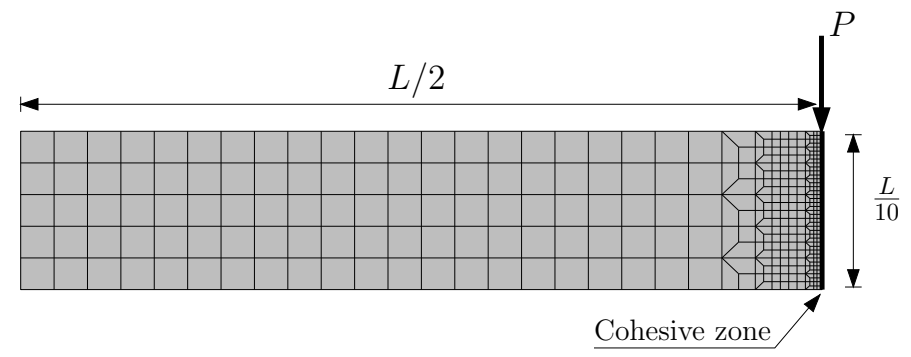

(b)

Figure 8. (a) Overview of model geometry and boundary conditions for the standard cemented slab on elastic solid foundation in ABAQUS ('CZM slab' model). (b) close-up of the slab region showing the cohesive zone mesh.

The CZM slab is modeled with 2-D plain strain elements (CPE4) in ABAQUS. A total of 1,400 elements are used to represent the elastic material, separated by pre-determined contact surfaces, representing the cohesive zone (10 mm size elements), in the vertical plane at the midbeam position. The subgrade soil is modeled with a total of 6,000 elements (CPE4). A close-up of the mesh in the slab region is shown in Figure 8 (b) and standard mechanical properties are given in Table 2.

Table 2. Material properties for single cement bound granular mixture slab on elastic foundation

\begin{tabular}{lccccccc}
\hline Material & $E_{c} / E_{s}(\mathrm{MPa})$ & $\nu(-)$ & $f_{t}(\mathrm{MPa})$ & $G_{F}(\mathrm{~N} / \mathrm{mm})$ & $k\left(\mathrm{~N} / \mathrm{mm}^{2}\right)$ & $\gamma(\mathrm{N})$ & $d(\mathrm{~m})$ \\
\hline CBGM & 15,000 & 0.20 & 0.80 & 0.035 & - & - & - \\
Soil & 100 & 0.35 & - & - & 0.0076 & $6.33 \cdot 10^{7}$ & 25 \\
\hline
\end{tabular}

\subsubsection{Solution technique and numerical characteristics}

To increase the efficiency of numerical simulations a conventional Newton-Raphson (N-R) method is implemented extended to handle displacement control, see e.g. Batoz and Dhatt (1979). The solver implemented sufficiently describes both pre- and post peak behaviour, whereas the potential snap-back load-displacement response is ignored.

Table 3. Solution technique and standard model parameters applied in the present study

\begin{tabular}{lc|lc}
\hline \multicolumn{2}{c|}{ Solver } & \multicolumn{2}{|c}{ Model parameters } \\
\hline Technique & $\mathrm{N}-\mathrm{R}$ & Cohesive zone width, $T_{0}(\mathrm{~mm})$ & 0.01 \\
Initial increment & 0.001 & Viscous damping parameter, $\mu(-)$ & $1 \times 10^{-5}$ \\
Maximum increment size & 0.01 & Bulk elements (elastic) & CPE4 $(2-\mathrm{D})$ \\
Minimum increment size & $1 \times 10^{-9}$ & Cohesive zone mesh $(\mathrm{mm})$ & 10 \\
\hline
\end{tabular}

Similarly the N-R method is selected for the purpose of analysis in ABAQUS. Based on preliminary convergence and sensitivity studies solution technique and standard model parameters selected for the present study are listed in Table 3. 


\section{Numerical model of a single hinge slab}

\subsection{Model geometry and analysis results}

Consider a single hinge slab, shown in Figure 9, consisting of cement bound granular mixture with standard dimensions $(L / h)$ of $4 \times 0.4 \mathrm{~m}^{2}$ supported by a two-parameter foundation, representing the semi-infinite elastic medium, and loaded by a concentrated force at midspan position. The soil adjacent to the slab can be modeled by extending the beam elements outside the slab to the length $d$ assigning a bending stiffness $E I$ close to zero. Alternatively an equivalent spring can be implemented at slab ends, representing the soil adjacent to the slab, i.e. $k_{\text {edge }}^{e q}=k \cdot \alpha$. Standard mechanical properties given in Table 2 are applied.

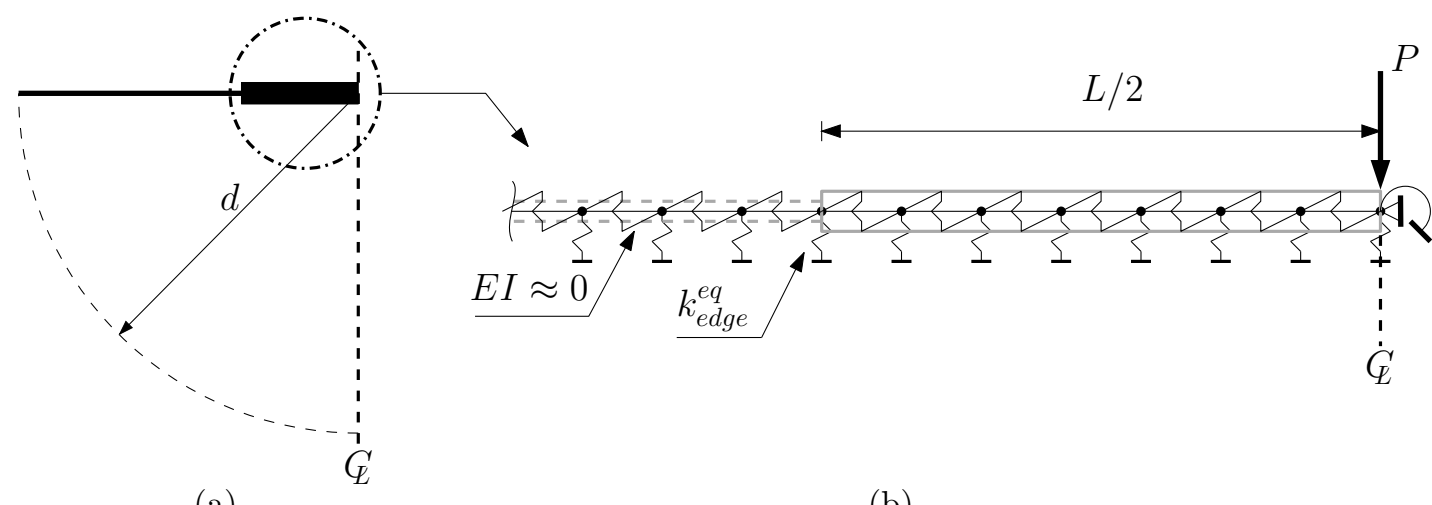

(a)

Figure 9. (a) Overview of model geometry and boundary conditions for the standard cemented slab on elastic solid foundation in ABAQUS ('CZM slab' model). (b) Model geometry, boundary conditions for the proposed cracked-hinge model resting on two-parameter foundation ('hinge slab' model).

Convergence test of the model is evaluated plotting the normalised peak-load $\eta$ and peak-load displacements $\zeta$ for different beam mesh densities, nel: 2-128, shown in Figure 10. It is observed that sufficient accuracy can be obtained with 64 elements, however, little is gained by increasing the number of elements from 32 to 64 . Element size of $0.1 \mathrm{~m}(n e l=40)$ is selected in subsequent analysis. Typical load-displacement response is shown in Figure 10 (b).

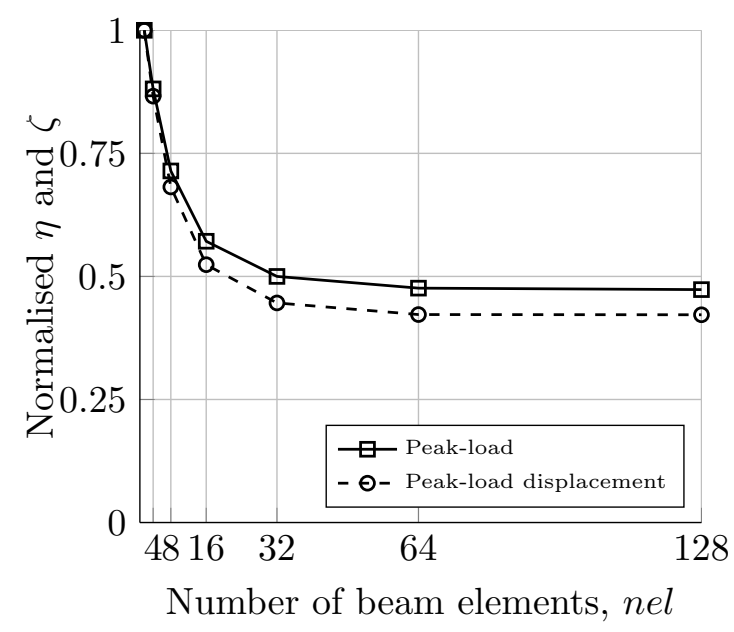

(a)

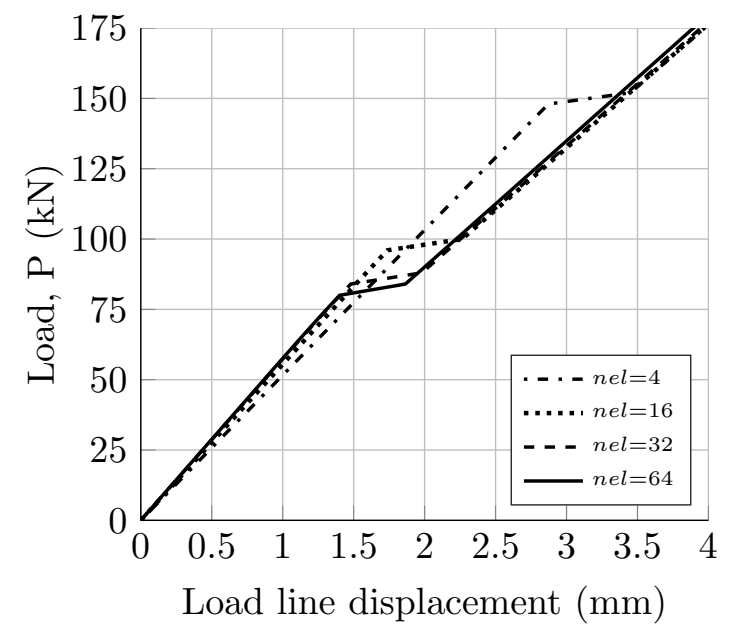

(b)

Figure 10. Convergence test: (a) Number of beam elements, nel: 2-128 (element size, elsz: 0.5-0.015625) versus the normalised first peak-load $\eta$ and peak-load displacements $\zeta$ applying foundation parameters for calibration method no. 1. (b) Load-displacement response.

In order to evaluate the fracture behavior of the hinge slab model, the load-crack mouth 
opening and load-crack length curve are plotted, shown in Figure 11 (a) and (b), respectively. To visualise the influence of the second parameter and to compare the two modeling techniques, the response for both hinge- and CZM slab resting on a Winkler foundation is shown for reference. Standard mechanical properties given in Table 2 are applied.

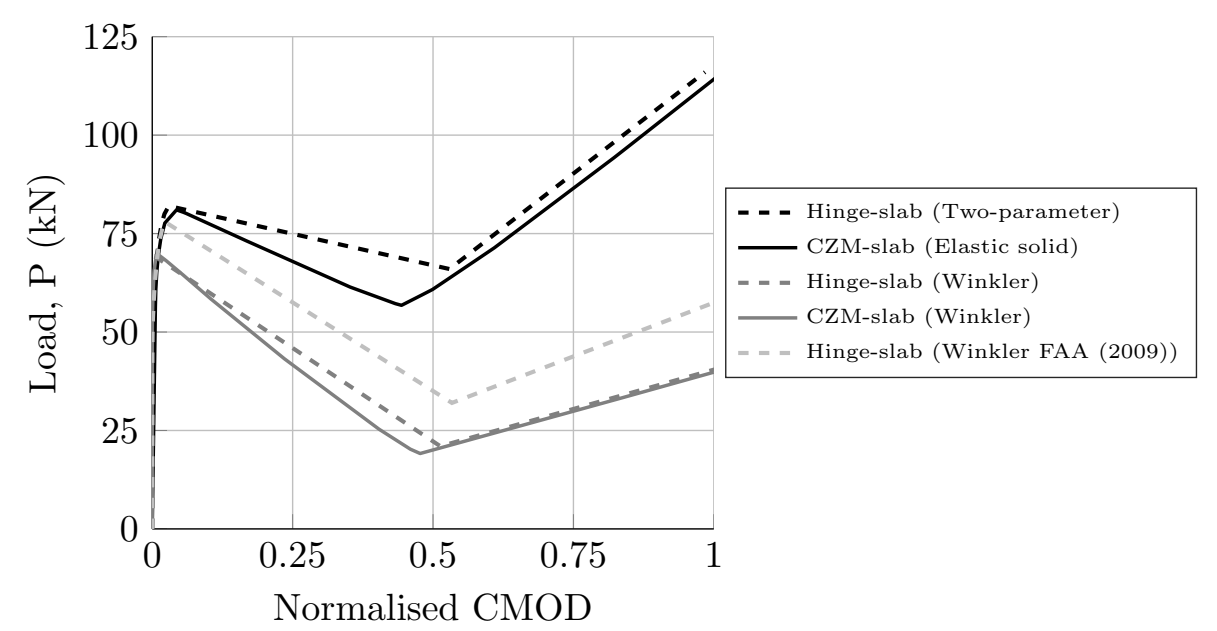

(a)

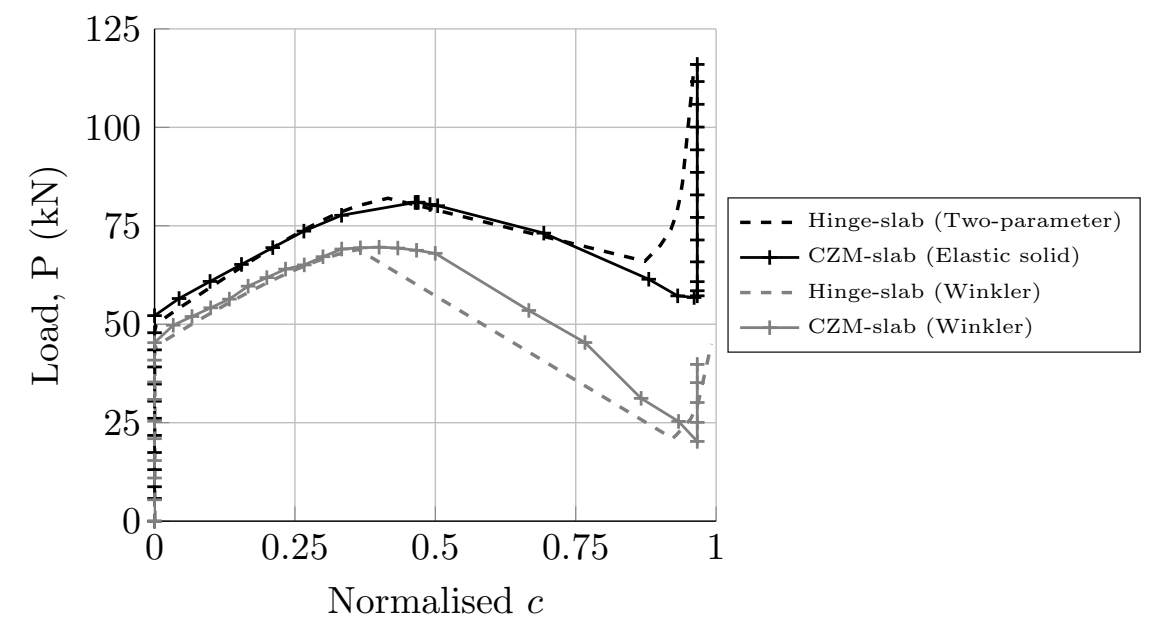

(b)

Figure 11. Comparing the structural response- and fracture behaviour of the hinge slab versus the CZM slab model in ABAQUS; (a) load-CMOD curve and (b) load-crack length curve. Where the crack length in the CZM slab model is taken as the progressive depth of damage initiation in the cohesive zone.

It is observed from Figure 11 (a) and (b) that reasonably good agreement between the hingeand CZM slab model can be obtained. Both models reflect the influence of the continuity in the soil comparing the two foundation types. It can also be shown that a closer prediction of the first peak-load can be obtained applying an empirical transfer function for converting the elastic soil properties to an apparent Winkler stiffness $k^{*}$ (FAA 2009). However, the post-peak response resemble that of the analytical Winkler foundation type due to the lack of continuity between springs.

The difference in first peak-load and peak-load displacement is app. $\pm 1 \%$ for the two model types. The two-parameter slab hinge model slightly overestimates the post-peak residual stiffness compared to the CZM slab model. Close to perfect fit is obtained between the two modeling techniques applying only a Winkler foundation as the supporting medium. It is also observed that the load level at unloading is higher for the hinge slab model compared to the CZM slab model. This tendency is observed for both foundation model types, and can partly be regarded 
as an effect of the difference in modeling technique. However, this difference is also related to the foundation type and contact behavior applied. This will be further discussed in the section below.

\subsection{Comparison of numerical modeling techniques}

A fundamental difference in the methodology between the CZM slab model and the hinge model is that the CZM slab model only considers one discrete crack at midspan position, whereas the effective stiffness concept adopted in the hinge model allows for multiple cracks along the beam. For the specific case a cracked zone of app. $1.0 \mathrm{~m}$ at the center of the slab have initiated and opened at first peak-load, shown in Figure 12 (a). Then, the crack at midspan progresses rapidly, meaning that the neighbouring cracks close.
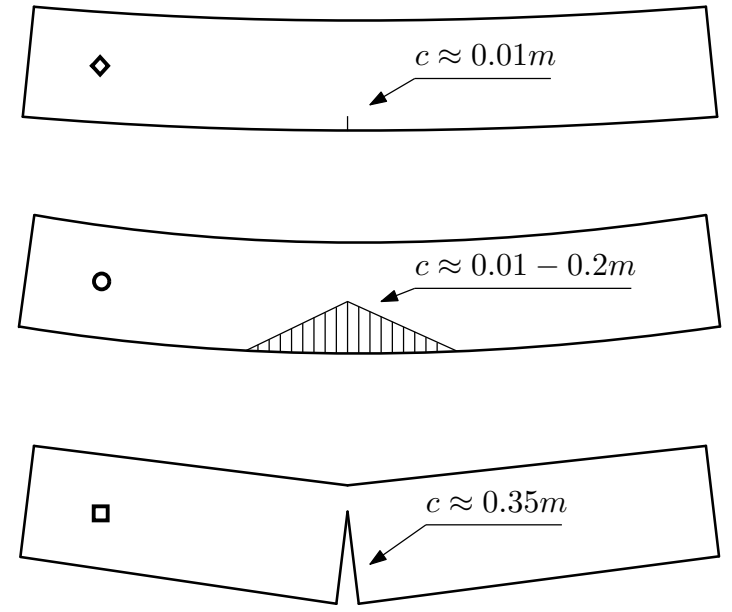

(a)

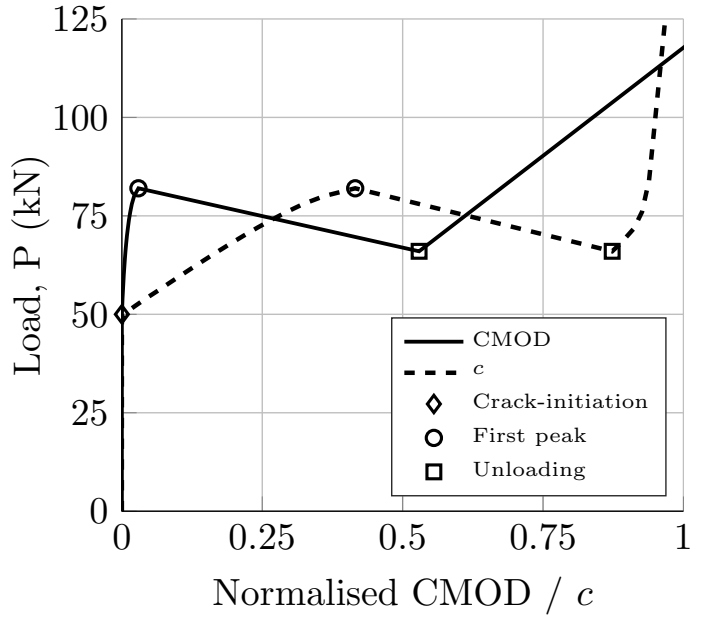

(b)

Figure 12. Development of crack width in slab for point of interest for the two-parameter model; (a) deformation of slab and crack lengths at points of interest and (b) points of interest plotted on the normalised load-CMOD and load-crack length curve.

Moreover, in the hinge model presented, increasing or decreasing the hinge rotation is modeled by the same equilibrium path. Thus, the hinge model does not have a unique unloading branch that models the closure of a previously opened crack. The cracks outside midspan position are small $(<1 \%)$ and do not influence the solution in the specific cases studied here.

A more significant influence on the model response can be found by studying the different soil models. Although the two-parameter model possess some of the characteristic features of continuous elastic solids, it is a simplification which cannot capture all complexities. Special care should be taken when selecting a representative load strip length for calibration of model parameters. This is exemplified plotting the vertical soil pressure below cemented slabs with different slenderness ratios (length/thickness ratio), shown in Figure 13.

The pressure will initially be distributed over the total slab length, as observed from Figure 13. As cracking is initiated and cracks propagate, there will be only minor changes in the overall vertical pressure profile, ignoring minor stress intensities below cracks. After crack propagation to a certain depth, the structure becomes unstable, resulting in unloading on the load-CMOD curve, see Figure 12 (b). This further results in a significant change in the vertical soil pressure and stresses localise around the open cracks, in this case at the midspan position. Moreover, a variation in vertical pressure, and especially the vertical reaction pressure along slab edges comparing different slab sizes is expected. The soil pressure under short thick slabs will resemble the stress distribution under a stiff plate, whereas the vertical reaction pressure along slab edges for larger size- and slender slabs will be small. 


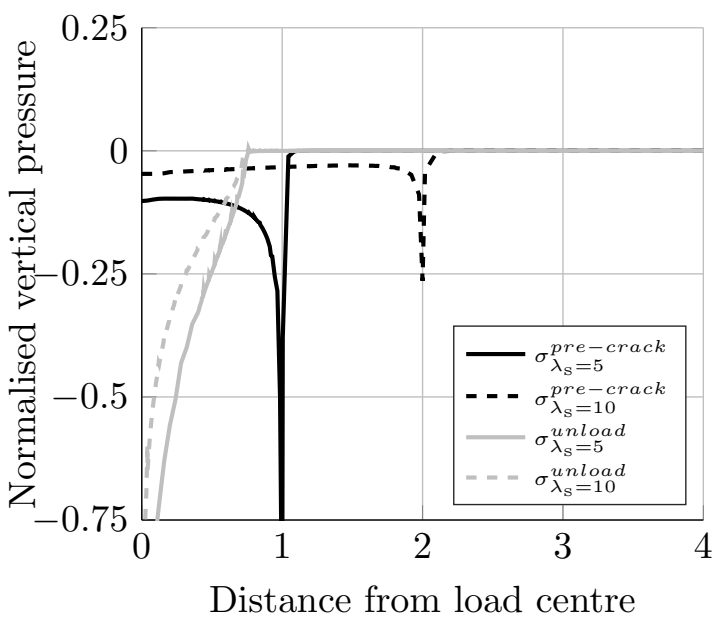

Figure 13. Vertical soil pressure of elastic solid under cemented slabs with slenderness ratio $\lambda_{s}=5$ and 10 before and after development of a single open crack below the loaded point, i.e. before 'first peak' and after 'unloading' in Figure 12 (b).

In order to capture both pre- and post-peak vertical pressure distributions in the twoparameter model considering typical slab lengths and thicknesses of app. 2.0-5.0 $\mathrm{m}$ and 0.15-0.45 $\mathrm{m}$, respectively, a fixed load strip length of $1.0 \mathrm{~m}$ is applied for calibration of foundation model parameters is recommended for the present problem.

The theoretical pressure distribution between the slab and the supporting elastic medium tend towards infinity at the perimeter, this off course, cannot occur in real soils. However, for cohesive soils the actual stress distribution will resemble the theoretical distribution. For granular soils stresses at the edges will be small because the bearing capacity at the surface of an unconfined granular material is small. In order to capture this behavior the methodology proposed could be extended using a 'Modified Pasternak model' consisting of two layers of vertical springs as proposed by Kerr (1964) allowing the slab to 'sink' into the supporting layer.

\section{Sensitivity studies}

\subsection{Effect of slab thickness and soil stiffness}

In linear elastic analysis of pavement structures, the slab- or layer thickness is increased for increasing design loads, to ensure both a relatively constant stress level in the soil foundation, avoiding any plastic deformation. However, as observed in Figure 14 this assumption is only valid as long as cracks are small as the thickness of the cemented slab has significant influence on the peak-load, but little influence on the post-peak response of the structure.

The stiffness- and peak-load of the structure increases with increasing thickness up to unloading. Then the structural response is mainly influenced by the stiffness of the soil foundation, as shown in Figure 15. 


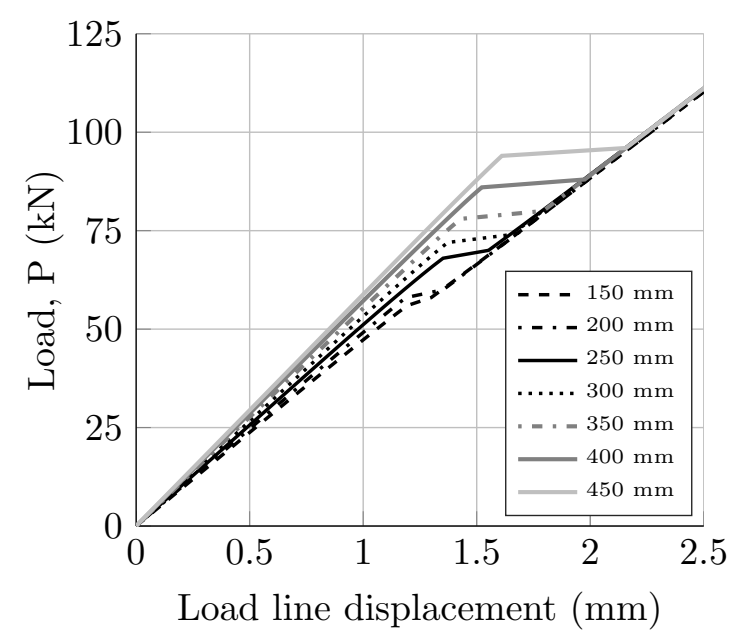

Figure 14. Influence of slab thickness on the load-displacement response. Thickness varying from 150 to $450 \mathrm{~mm}$ keeping length constant $(3 \mathrm{~m})$.

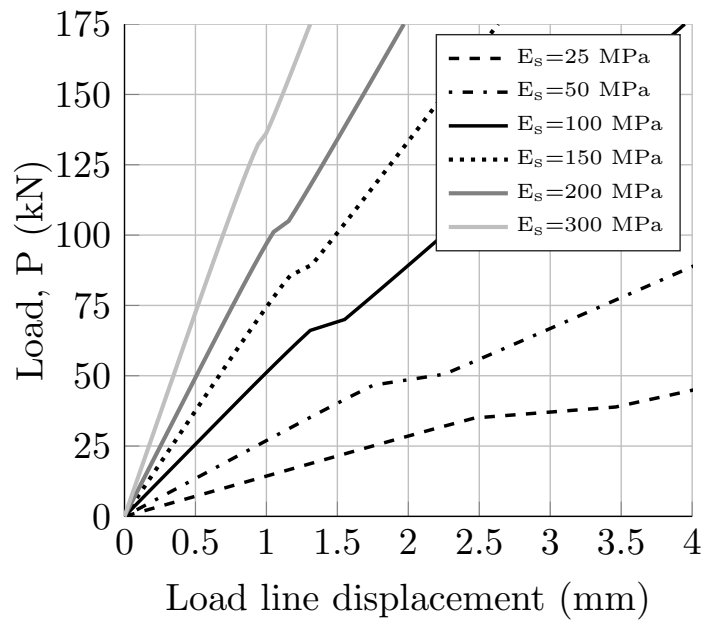

Figure 15. Influence of soil stiffness on the load-displacement response. Young's modulus $E_{s}$ varying from 25-300 MPa.

As expected increasing stiffness of the soil results in increasing peak-load and post-peak stiffness, as shown in Figure 15. It can also be concluded that the soil stiffness has a more significant influence on the peak-load than the thickness of slabs. The influence of slab size is found to be small as only crack propagation in one direction is considered here.

\subsection{Effect of tensile strength and fracture energy}

Considering a simple linear softening law, it is obvious that the two main material parameters influencing the fracture process of the quasi-brittle material are the tensile strength and the fracture energy. Both tensile strength and fracture energy influence the peak-load and peak-load displacement, as shown in Figure 16 (a) and (b), respectively.

It should be noted that the influence of tensile strength will be reduced compared to the fracture energy in three-dimensional simulations, as the out-of-plane crack propagation significantly influences the load-displacement response (Gaedicke and Roesler 2009; Aure and Ioannides 2012; Skar and Poulsen 2015). 


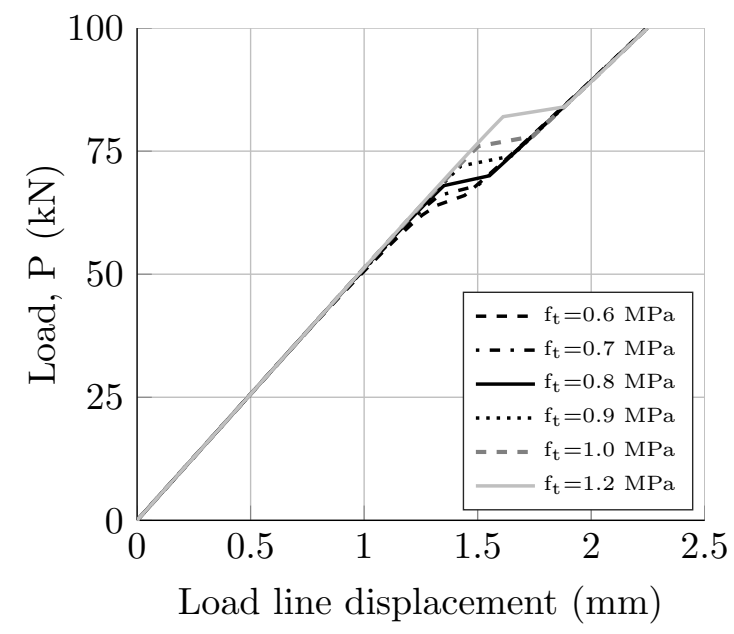

(a)

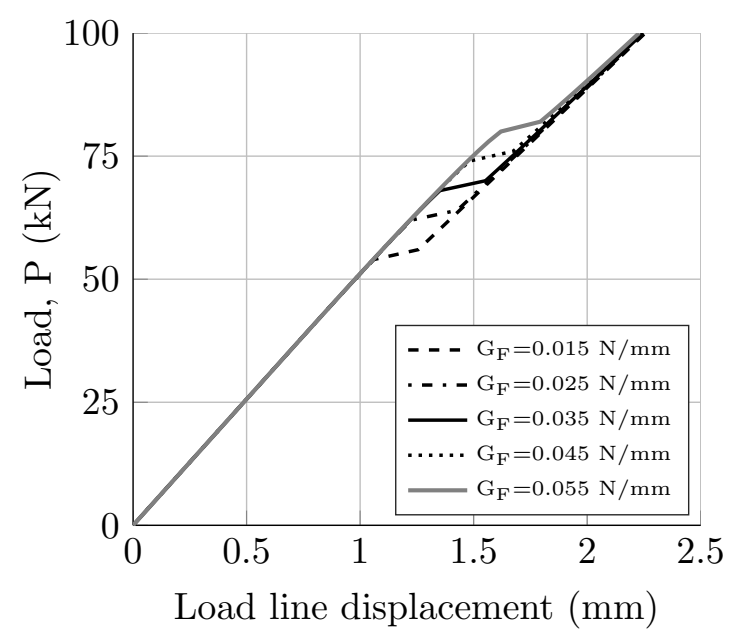

(b)

Figure 16. Influence of fracture properties on the load-displacement response: (a) tensile strength $f_{t}$ varying from 0.6-1.2 $\mathrm{MPa}$ and (b) fracture energy $G_{F}$ varying from $0.015 \mathrm{~N} / \mathrm{mm}$ to $0.055 \mathrm{~N} / \mathrm{mm}$.

\subsection{Effect of load configuration and position}

Realistic concrete- and composite pavement systems are subjected to different loading conditions. In order to demonstrate the applicability of the proposed method to evaluate different load cases, the load-displacement response for typical load configurations and positions is plotted, shown in Figure 17.

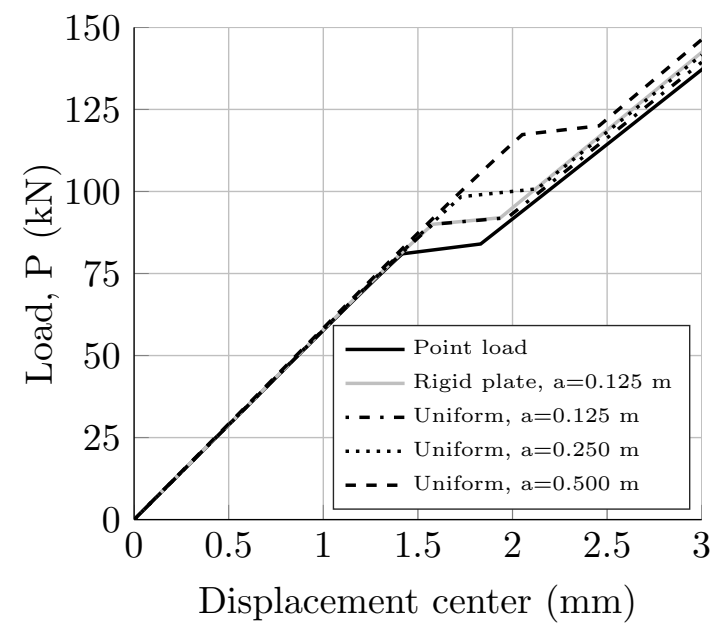

(a)

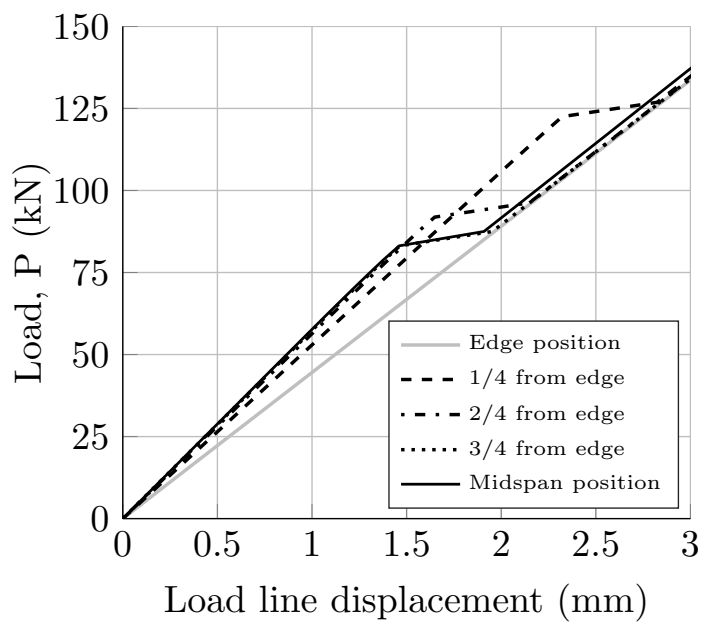

(b)

Figure 17. Influence of load configuration and position on load-displacement response: (a) typical load configurations, e.g. rigid and uniformly distributed loads with varying load strip length $a$ and (b) slab loaded at edge, midspan and intermediate positions.

It is observed from Figure 17 (a) that increasing load strip length results in increasing peakload, as expected. For the rigid plate load, modeled as two point loads at each end of the loaded strip, one crack develop at each edge. However, for the two-dimensional problem considered here, this has little influence on the structural response compared to a uniformly distributed load. From Figure 17 (b) it is observed that the peak-load increase for increasing distance from midspan position, whereas the stiffness decrease. Loading at midspan and intermediate positions result in one crack opening and progressing after the first peak, whereas no cracks develop in case of edge loading. 


\section{Structural example}

In order to demonstrate the applicability of the numerical hinge to predict a realistic crack pattern an infinite slab $(h=0.4 \mathrm{~m})$ resting on Winklers foundation $(k=0.0233 \mathrm{MPa} / \mathrm{mm})$ and loaded by a concentrated force at midspan position is considered. Slab displacements and internal forces decline relatively fast to zero as the distance from the load increases. Accordingly, a finite slab of $10 \mathrm{~m}(n e l=100)$ may be replacing the infinite beam. Standard material properties for the cemented material given in Table 2 is applied.

The numerical results are compared to the yield-line theory following the methodology proposed by Baumann and Weisgerber (1983). The deformation of the slab during loading, i.e. before first peak, before second peak and after the second peak, is shown in Figure 18 (a). Load-displacement curves for the three models are plotted in Figure 18 (b).

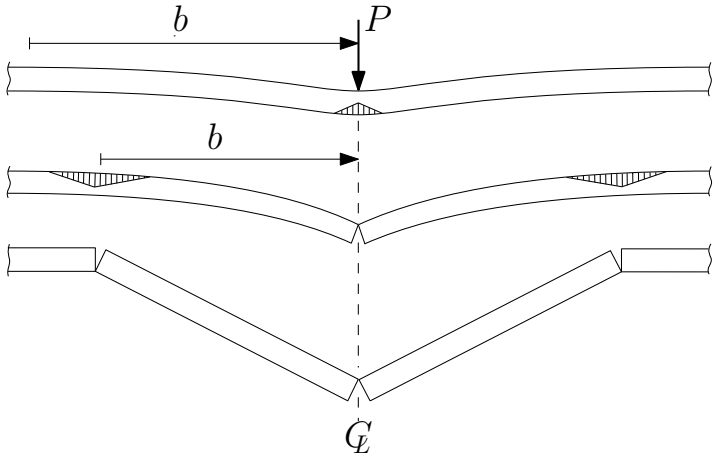

(a)

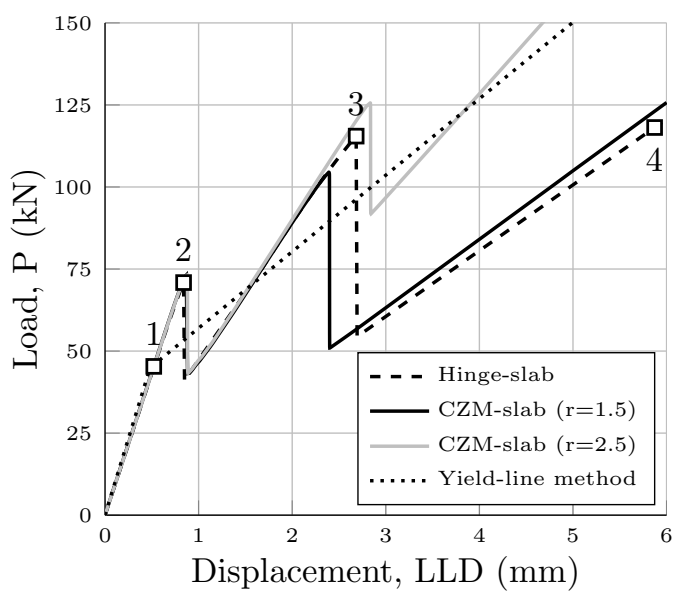

(b)

Figure 18. Comparison between modeling techniques for infinite slab: (a) Deformation of the slab during loading: before first peak, after first peak and after second peak. (b) CZM slab (solid line), hinge slab model (dashed line) and the yield-line method (dotted line) reported in Baumann and Weisgerber (1983).

It is observed from Figure 18 (b) that the first peak in the CZM slab model and the hinge slab model is app. 73 and $71 \mathrm{kN}$, respectively. Moreover, it is observed that the kink point on the yield-line curve is app. $45 \mathrm{kN}$, complying well with the results reported by Meda (2003). Moreover, it is found that the distance between yield-lines $r=2.534 \mathrm{~m}$ in the yield line method is approximately equal to the distance, $b$, from the negative peak moments to the crack at midspan in the hinge slab model at crack-initiation (load level 1), shown in Figure 19 (a). Then, according to the CZM slab and hinge slab model the load continues to increase as the crack propagates before reaching the ultimate moment capacity of app. $33.3 \mathrm{kNm}$ (load level 2), shown in Figure 19 (b).

The crack at midspan then unloads, before the load continues to increase, resulting in two top-down cracks initiating app. $\pm 1.5 \mathrm{~m}$ from the midspan position. Then, these cracks grow rapidly to the negative ultimate moment capacity of app. $-33.3 \mathrm{kNm}$ (load level 3 ), shown in Figure 19 (c), resulting in the second peak on the load-displacement curve. Finally, the post-peak residual stiffness of the structure is reached (load level 4) with the moment distribution shown in Figure 19 (d).

The distance between cohesive zones in the CZM slab model is set to $1.5 \mathrm{~m}$ based on simulations with the hinge slab model. This results in a good fit between the two methods as shown in 18 (b). The responses of the two methods are identical up to the second peak of the CZM slab model. Then, the load in the hinge slab model continues to increase due to the stiffness and stress redistribution in the hinge slab. The second peak in the models occur at app. 105 and 115 $\mathrm{kN}$, respectively. The difference in response between models is small up to first peak as cracks 


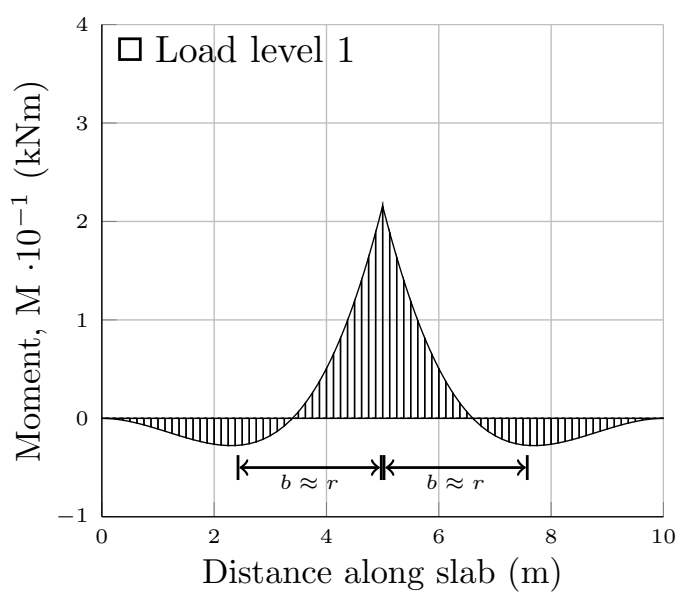

(a)

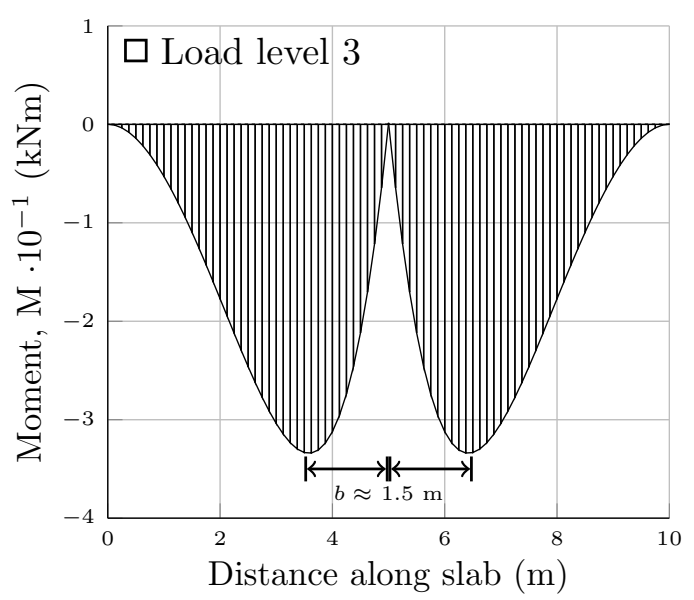

(c)

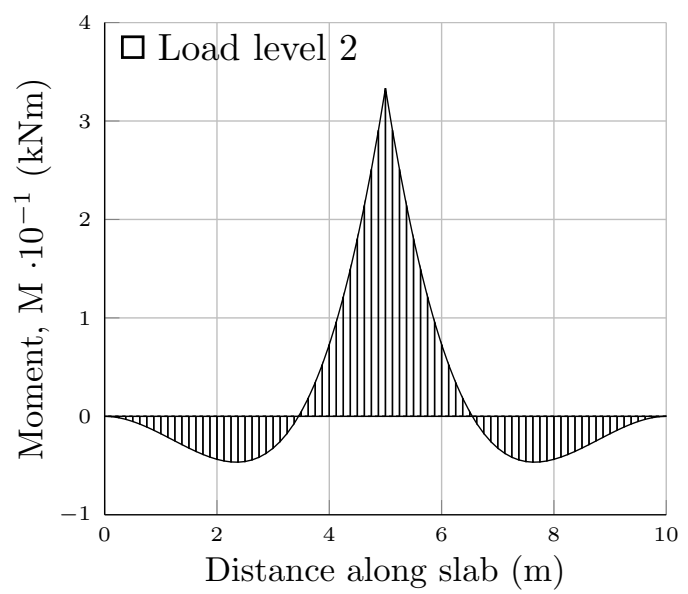

(b)

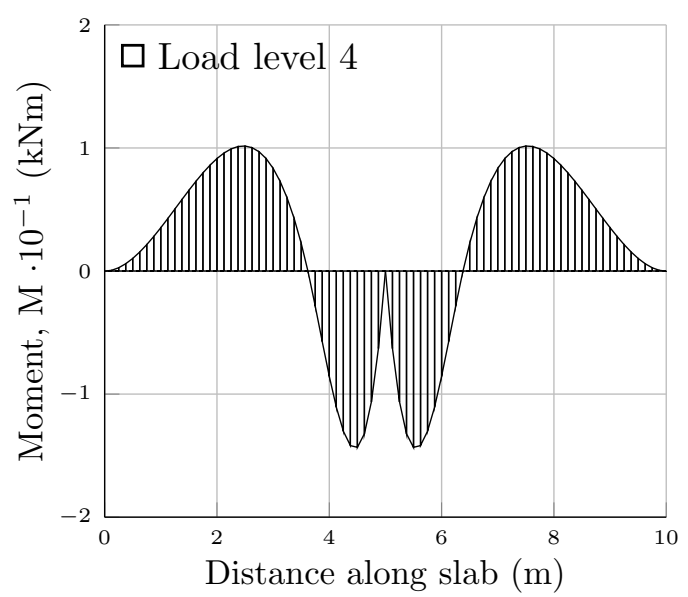

(d)

Figure 19. Predicting distance between cracks showing the moment in the hinge slab model at four different phases: (a) crack initiation, (b) first peak, (c) second peak and (d) after full crack propagation of top-down cracks, also visualising the distance between cracks equivalent to $r$ in the yield line method.

localise to one crack below the loaded point. Then top-down cracks at a distance $b$ develop, however, these are smeared over a larger area, resulting in evolving stress redistribution in the slab during crack propagation. This phenomena cannot be captured by the CZM slab model. Thus a lower second peak load is predicted with the CZM slab model. Increasing the length between cohesive zones in the CZM model would result in increasing second-peak and post-peak stiffness.

The present example highlights the practical use of the model proposed; the hinge slab being able to predict the stress redistribution and stiffness during crack development. This results in a precise prediction the crack-opening and the distance between cracks that finally localise and propagate through the thickness of the slab.

\section{Conclusion}

The use of a cohesive cracked-hinge model for simulating the fracture in one-way slab on grade structures has been investigated showing good performance.

Comparing numerical and experimental results for four point bending beam tests show that good agreement is obtained with the hinge model. It is also found that good agreement is 
obtained for both structural and fracture behavior of slab on grade structures, comparing the hinge model with the more conventional cohesive zone model. The adaptive hinge proved to adequately predict the distance between cracks compared to the yield-line theory. Moreover, peak-loads and structural response comply well with other results reported in the literature. The hinge model has the advantage that it is numerically robust, resulting in fast simulations and few convergence issues.

It can be shown that the two-parameter foundation model and the calibration methodology proposed is an efficient tool which realistically captures the response of the elastic medium below the slab. The calibration methodology is consistent and model parameters are determined on a rational basis. Moreover, the foundation model applied is applicable for implementation in commercial codes applying discrete spring elements and/or connectors, making the methodology an attractive alternative for design engineers.

Slab thickness and soil stiffness proved to be important parameters. The peak-load is highly influenced by thickness of the slab, whereas the soil stiffness proved to be a main controlling parameter of both pre- and post-peak response of the structure. Furthermore, it can be concluded that the fracture process is more affected by the fracture energy than by the tensile strength.

The present paper demonstrates the use of a cohesive crack-hinge model resting on a twoparameter foundation to describe the fracture behavior of cemented slab on grade structures. Although a simplified two-dimensional problem is investigated here, the results obtained are encouraging, showing that the methodology is applicable for practical use. Moreover, a general and consistent format is applied making the methodology well suited for further development. 


\section{References}

ABAQUS, 2013. Analysis users manual, Version 6.13-1. [online] [????].

Aure, T.W. and Ioannides, A.M., 2012. Numerical analysis of fracture process in pavement slabs. Canadian Journal of Civil Engineering, 39 (5), 506-514.

Aure, T.W. and Ioannides, A.M., 2015. Fracture analysis of aggregate interlock jointed slabs-on-grade. Construction and Building Materials, 77 (0), 340 - 348.

Batoz, J.L. and Dhatt, G., 1979. Incremental displacement algorithms for nonlinear problems. International Journal for Numerical Methods in Engineering, 14 (8), 1262-1267.

Baumann, R.A. and Weisgerber, F.E., 1983. Yield-line analysis of slabs-on-grade. Journal of Structural Engineering, 109 (7), 1553-1568.

Buratti, N., Mazzotti, C., and Savoia, M., 2011. Post-cracking behaviour of steel and macro-synthetic fibre-reinforced concretes. Construction and Building Materials, 25 (5), 2713-2722.

Carpinteri, A. and Corrado, M., 2011. Upper and lower bounds for structural design of RC members with ductile response. Engineering Structures, 33 (12), 3432-3441.

Castel, A., Vidal, T., and François, R., 2011. Finite-element modeling to calculate the overall stiffness of cracked reinforced concrete beams. Journal of Structural Engineering, 138 (7), 889-898.

Colasanti, R.J. and Horvath, J.S., 2010. Practical subgrade model for improved soil-structure interaction analysis: software implementation. Practice Periodical on Structural Design and Construction, 15 (4), 278-286.

Cook, R.D., et al., 2007. Concepts and applications of finite element analysis. John Wiley \& Sons.

Evangelista, Francisco, J., Roesler, J.R., and Proena, S.P., 2013. Three-dimensional cohesive zone model for fracture of cementitious materials based on the thermodynamics of irreversible processes. Engineering Fracture Mechanics, 97, 261-280.

FAA, 2009. Advisory Circular (AC) 150/5320-6E. [online] [????].

Filonenko-Borodich, M., 1940. Some approximate theories of the elastic foundation. Uchenyie Zapiski Moskovskogo Gosudarstvennogo Universiteta Mekhanica, 46, 3-18.

Flamant, A., 1892. Compt. Rend. 114. (114), 1465.

Gaedicke, C., Roesler, J., and Evangelista, F., 2012. Three-dimensional cohesive crack model prediction of the flexural capacity of concrete slabs on soil. Engineering Fracture Mechanics, 94, 1-12.

Gaedicke, C. and Roesler, J., 2009. Fracture-based method to determine the flexural load capacity of concrete slabs. FAA COE Rep, (31).

Hetenyi, M., 1966. Beams and plates on elastic foundations and related problems. Appl. Mech. Rev, 19 (2), 95-102.

Hetényi, M., 1946. Beams on elastic foundation: theory with applications in the fields of civil and mechanical engineering. University of Michigan.

Hillerborg, A., Modéer, M., and Petersson, P.E., 1976. Analysis of crack formation and crack growth in concrete by means of fracture mechanics and finite elements. Cement and concrete research, 6 (6), $773-781$.

Horvath, J., 2002. Soil-structure interaction research project: Basic SSI concepts and applications overview. Manhattan College School of Engineering, Bronx, New York, USA.

Ioannides, A., Thompson, M., and Barenberg, E., 1985. Finite element analysis of slabs-on-grade using a variety of support models. In: Third International Conference on Concrete Pavement Design and Rehabilitation, 1 Volume.

Ioannides, A.M., 2006. Concrete pavement analysis: the first eighty years. International Journal of Pavement Engineering, 7 (4), 233-249.

Ioannides, A.M., Peng, J., and Swindler Jr, J.R., 2006. ABAQUS model for PCC slab cracking. International Journal of Pavement Engineering, 7 (4), 311-321.

Kerr, A.D., 1964. Elastic and viscoelastic foundation models. Journal of Applied Mechanics, 31 (3), 491498.

Kerr, A.D., 1965. A study of a new foundation model. Acta Mechanica, 1 (2), 135-147.

Khazanovich, L., 2003. Finite element analysis of curling of slabs on Pasternak foundation. In: 16th ASCE Engineering Mechanics Conference, July, 16-18.

Khazanovich, L. and Ioannides, A.M., 1993. Finite element analysis of slabs-on-grade using higher order subgrade soil models. In: Airport Pavement Innovations Theory to Practice, sept 8-10, 1993, Vicksburg, Mississippi, USA. 
Khazanovich, L. and Ioannides, A.M., 1994. Structural analysis of unbonded concrete overlays under wheel and environmental loads. Transportation Research Record, (1449).

Kwak, H.G. and Kim, S.P., 2002. Nonlinear analysis of RC beams based on moment-curvature relation. Computers \&f structures, 80 (7), 615-628.

Kwak, H.G. and Kim, S.P., 2010. Simplified monotonic moment-curvature relation considering fixed-end rotation and axial force effect. Engineering Structures, 32 (1), 69-79.

Loof, H., 1965. The theory of the coupled spring foundation as applied to the investigation of structures supported on soil. HERON, 13 (3), 1965.

Meda, A., 2003. Design methods for slabs on grade in fiber reinforced concrete. System-based Vision for Strategic and Creative Design, Vols 1-3, 1413-1418.

Meda, A., Plizzari, G.A., and Riva, P., 2004. Fracture behavior of SFRC slabs on grade. Materials and StructuresS, 37 (270), 405-411.

Murthy, A.R., et al., 2013. Bilinear tension softening diagrams of concrete mixes corresponding to their size-independent specific fracture energy. Construction and Building Materials, 47, 1160-1166.

NCHRP, 2003. Guide for mechanistic-empirical design of new and rehabilitated pavement structures: Structural response modeling of rigid pavements - Appendix QQ. National Cooperative Highway Research Program, Transportation Research Board, National Research Council, Washington, DC.

Olesen, J.F., 2001a. Cracks in reinforced FRC beams subject to bending and axial load. Fracture mechanics of concrete structures, vols 1 and 2, 1027-1033.

Olesen, J.F., 2001b. Fictitious crack propagation in fiber-reinforced concrete beams. Journal of Engineering Mechanics, 127 (3), 272-280.

Olesen, J.F., Østergaard, L., and Stang, H., 2006. Nonlinear fracture mechanics and plasticity of the split cylinder test. Materials and structures, 39 (4), 421-432.

Pasternak, P., 1954. On a new method of analysis of an elastic foundation by means of two constants [Gosudarstvennoe Izdatelstvo Literaturi po Stroitelstvu I Arkhitekture]. Moscow: USSR.

Reissner, E., 1958. A note on deflections of plates on a viscoelastic foundation. Journal of Applied Mechanics, 25 (1), 144-145.

Skar, A. and Poulsen, P.N., 2015. 3-D cohesive finite element model for application in structural analysis of heavy duty composite pavements. Construction and Building Materials, 101, Part 1, 417 - 431.

Timoshenko, S., Goodier, J., and Abramson, H.N., 1970. Theory of elasticity. Journal of Applied Mechanics, 37, 888 .

Ulfkjær, J.P., Krenk, S., and Brincker, R., 1995. Analytical model for fictitious crack propagation in concrete beams. Journal of Engineering Mechanics, 121 (1), 7-15.

Visintin, P., et al., 2012. A mechanics solution for hinges in RC beams with multiple cracks. Engineering Structures, 36, 61-69.

Vlasov, V.Z., 1966. Beams, plates and shells on elastic foundations. Israel Program for Scientific Translations, Jerusalem.

Walter, R., et al., 2005. Wedge splitting test for a steel-concrete interface. Engineering fracture mechanics, 72 (17), 2565-2583.

Wardeh, G. and Ghorbel, E., 2015. Prediction of fracture parameters and strain-softening behavior of concrete: effect of frost action. Materials and Structures, 48 (1-2), 123-138.

Winkler, E., 1868. Die lehre von der elasticitaet und festigkeit. Verlag Dominicus, Prag.

Yeo, R., 2008. The development and evaluation of protocols for the laboratory characterisation of cemented materials. AP-T101/08.

Zokaei-Ashtiani, A., Carrasco, C., and Nazarian, S., 2014. Finite element modeling of slab-foundation interaction on rigid pavement applications. Computers and Geotechnics, 62, 118-127.

Zokaei-Ashtiani, A., et al., 2015. Impact of different approaches to modelling rigid pavement base layers on slab curling stresses. International Journal of Pavement Engineering, 1-9. 


\section{Appendix A. Expressions for calibration of two-parameter model}

\section{A.1 Two-parameter model}

Expressions for points of interest for the two-parameter model can be found from (11), i.e.

$$
\begin{aligned}
w(0) & =\frac{q}{k}\left[1-e^{-\beta a}\right] \\
w(a) & =\frac{q}{2 k}\left[1-e^{-2 \beta a}\right] \\
w^{\prime \prime}(0) & =\frac{\beta^{2} q}{k}\left[e^{-\beta a}\right]
\end{aligned}
$$

where $w(0)$ is the vertical displacement at the center of the loaded area, $w(a)$ is the vertical displacement at the edge of the loaded area and $w^{\prime \prime}(0)$ is the curvature at the center of the loaded area.

\section{A.2 Elastic continuum model}

Expressions for points of interest for the continuum model can be found from (13), i.e.

$$
\begin{aligned}
w(0) & =\frac{2 q a}{\pi E}\left[2 \ln \left(\frac{d}{|a|}\right)+(1-\nu)\right] \\
w(a) & =\frac{2 q a}{\pi E}\left[2 \ln \left(\frac{d}{|2 a|}\right)+(1-\nu)\right] \\
w^{\prime \prime}(0) & =-\frac{4 q}{\pi E a}
\end{aligned}
$$

where $w(0)$ is the vertical displacement at the center of the loaded area, $w(a)$ is the vertical displacement at the edge of the loaded area and $w^{\prime \prime}(0)$ is the curvature at the center of the loaded area.

\section{Appendix B. Derivation of hinge tangent stiffness matrix}

For the linear elastic Phase 0 , the hinge tangent stiffness matrix is given as

$$
\mathbf{D}_{t}^{0}=\left[\begin{array}{cc}
h t E_{c} & 0 \\
0 & \frac{1}{12} t h^{3} E_{c}
\end{array}\right]
$$

The constituents of the hinge tangent stiffness matrix in (20) are obtained from the sectional forces $N$ and $M$ in (7a) and (7b), respectively.

Utilizing the following relations for the relevant part of the integral corresponding to $0<w \leq$ $w_{c}$

$$
\frac{\partial \sigma_{c}}{\partial \bar{\varepsilon}_{0}}=E_{c} \frac{a s}{E_{c}+a s}, \frac{\partial \sigma_{c}}{\partial \bar{\kappa}}=E_{c} \frac{a s}{E_{c}+a s} y
$$


Here the parameters $\alpha=\frac{a s}{E_{c}+a s}$ and $E_{c c}=E_{c} \alpha$ is introduced, where the latter represents the reduced stiffness of the cracked part of the hinge. The full hinge tangent stiffness matrix for Phase $I$ and $I I$ can then be established

$$
\begin{aligned}
d N & =t \int_{-h / 2}^{h / 2-c} E_{c}\left(d \bar{\varepsilon}_{0}+d \bar{\kappa} y\right) d y+t \int_{h / 2-c}^{h / 2} E_{c c}\left(d \bar{\varepsilon}_{0}+d \bar{\kappa} y\right) d y \\
& =D_{t}(1,1) d \bar{\varepsilon}_{0}+D_{t}(1,2) d \bar{\kappa} \\
d M & =t \int_{-h / 2}^{h / 2-c} y E_{c}\left(d \bar{\varepsilon}_{0}+d \bar{\kappa} y\right) d y+t \int_{h / 2-c}^{h / 2} y E_{c c}\left(d \bar{\varepsilon}_{0}+d \bar{\kappa} y\right) d y \\
& =D_{t}(2,1) d \bar{\varepsilon}_{0}+D_{t}(2,2) d \bar{\kappa}
\end{aligned}
$$

which by solving the differential equation yields

$$
\begin{aligned}
D_{t}(1,1) & =E_{c} t\left(\left(y_{2}-y_{1}\right)+\left(y_{3}-y_{2}\right) \alpha\right) \\
D_{t}(1,2) & =E_{c} t\left(\frac{1}{2}\left(y_{2}-y_{1}\right)\left(y_{1}+y_{2}\right)+\frac{1}{2}\left(y_{3}-y_{2}\right)\left(y_{2}+y_{3}\right) \alpha\right) \\
D_{t}(2,1) & =D_{t}(1,2) \\
D_{t}(2,2) & =E_{c} t\left(\frac{1}{3}\left(y_{2}-y_{1}\right)\left(y_{1}^{2}+y_{2}^{2}+y_{1} y_{2}\right)\right. \\
& \left.+\frac{1}{3}\left(y_{3}-y_{2}\right)\left(y_{2}^{2}+y_{3}^{2}+y_{2} y_{3}\right) \alpha\right) \\
\mathbf{D}_{t}^{I, I I} & =\left[\begin{array}{l}
D_{t}(1,1) D_{t}(1,2) \\
D_{t}(2,1) D_{t}(2,2)
\end{array}\right]
\end{aligned}
$$

where $y_{1}, y_{2}$ and $y_{3}$ is the distances depicted on Figure 2 .

Contribution from a reinforcement rebar can alternatively be included in the beam-hinge tangent stiffness matrix as

$$
\mathbf{D}_{t}^{I, I I}=\left[\begin{array}{cc}
D_{t}(1,1)+E_{s} A_{s} & D_{t}(1,2)+E_{s} A_{s} y_{s} \\
D_{t}(2,1)+E_{s} A_{s} y_{s} & D_{t}(2,2)+E_{s} A_{s} y_{s}^{2}
\end{array}\right]
$$

where $A_{s}$ is the area of the rebar, $E_{s}$ the Young's modulus of the steel and $y_{s}$ is the position of the rebar. 